\title{
Numerical methods for the detection of phase defect structures in excitable media
}

\author{
Desmond Kabus ${ }^{1,2,3}$, Louise Arno ${ }^{1,3}$, Lore Leenknegt ${ }^{1,3}$, Alexander V. Panfilov ${ }^{4,5,6}$, \\ Hans Dierckx ${ }^{1,3^{*}}$
}

1 Department of Mathematics, KU Leuven Campus Kortrijk, Belgium

2 Laboratory of Experimental Cardiology, Leiden University Medical Center, Leiden, The Netherlands

3 iSi Health, KU Leuven Institute of Physics-based Modeling for In Silico Health, Leuven, Belgium

4 Department of Physics and Astronomy, Ghent University, Ghent, Belgium

5 Laboratory of Computational Biology and Medicine, Ural Federal University, Ekaterinburg, Russia

6 World-Class Research Center "Digital biodesign and personalized healthcare", Sechenov University, Moscow, Russia

*h.dierckx@kuleuven.be

\section{Abstract}

Electrical waves that rotate in the heart organize dangerous cardiac arrhythmias. Finding the region around which such rotation occurs is one of the most important practical questions for arrhythmia management. For many years, the main method for finding such regions was so-called phase mapping, in which a continuous phase was assigned to points in the heart based on their excitation status and defining the rotation region as a point of phase singularity. Recent analysis, however, showed that in many rotation regimes there exist phase discontinuities and the region of rotation must be defined not as a point of phase singularity, but as a phase defect line. In this paper we use this novel methodology and perform comparative study of three different phase definitions applied to in-silico data and to experimental data obtained from optical voltage mapping experiments on monolayers of human atrial myocytes. We introduce new phase defect detection algorithms and compare them with those that appeared in literature already. We find that the phase definition is more important than the algorithm to identify sudden spatial phase variations. Sharp phase defect lines can be obtained from a phase derived from local activation times observed during one cycle of arrhythmia. Alternatively, similar quality can be obtained from a reparameterization of the classical phase obtained from observation of a single timeframe of transmembrane potential. We found that the phase defect line length was $(35.9 \pm 6.2) \mathrm{mm}$ in the Fenton-Karma model and $(4.01 \pm 0.55) \mathrm{mm}$ in cardiac human atrial myocyte monolayers. As local activation times are obtained during standard clinical cardiac mapping, the methods are also suitable to be applied to clinical datasets. All studied methods are publicly available and can be downloaded from an institutional web-server. 


\section{Introduction}

The heart is a self-organizing dynamical system for which the mechanical contraction is regulated by waves of electrical activation travelling through the cardiac wall. During cardiac arrhythmia complex electrical patterns emerge that often result into a rotating pattern, either circling around an obstacle or around its own wave back 1,2]. These vortices are also known as rotors, or spiral waves in two dimensions (2D), or scroll waves in three dimensions (3D).

After the experimental observation of such structures in animal hearts during ventricular tachycardia [3], it was conjectured that rotors can sustain several heart rhythm disorders. However, the precise dynamics of rotors, the structure of the rotor core and the most efficient manner to remove them from the heart remain incompletely understood.

A quantitative description of spiral wave motion requires to localize it in space. In first approximation, the region around which the rotor revolves is called the spiral wave core. The location of a spiral wave at a given time can be further narrowed down to a single point, usually called the spiral wave tip. Different methods exist to define the tip, e.g. as the point where wave front and wave back merge [4, as the point on a line of constant voltage that does not change instantaneously, as an intersection between two isolines of different variables [5] or as a point singularity of the activation phase [2,6].

By following the tip position of a single spiral wave over time, a disc-like or star-like shape emerges, known as the spiral wave core. Different types of cores have been observed [7, and the non-circular cores are referred to as meandering cores. Among the meandering cores, simulations of detailed ionic models for cardiac tissue typically show so-called linear cores, as depicted in Fig 1 .

The linear-core regime arises in systems with long action potential duration (APD): If the tip is next to a region of refractory tissue, it will follow this interface until meeting a point where the tissue has recovered. As a result, the tip moves along an almost straight line, interleaved with turning points. These dynamics have not only been observed in simulations, but were also reported in experiments 8 and clinical observations 9 in the form of a line of conduction block. For this reason it is important to elucidate the spatial distribution of the core of the rotor.

In 3D, a spiral wave or rotor becomes a structure called a scroll wave. Within the scroll wave, the collection of spiral wave tips forms a filament curve [10]. In numerical simulations with linear cores and a few experimental observations [8], the straight segment in the linear core extends to a ribbon-like filament 8 . However, these spatially extended filaments have not been substantially included in theory development, as was done for circular-core filaments [11.

Recent works 12,13 have proposed to treat linear cores fundamentally different from circular cores. Specifically, classical phase analysis 2, 6] of cardiac activation patterns assumes that there is a phase singularity (PS) located near the spiral wave tip. However, when the wave front reaches a part of tissue that is for instance not fully recovered yet, a so-called conduction block will form. On both sides of this conduction block line, there is a different phase, such that the authors of 12 13] argue that the phase representation resembles more a branch cut or phase defect line (PDL) than a point singularity. To make the distinction between phase defects and point singularities could have potential clinical use, as current analysis methods in simulation, experiment and of 
bioRxiv preprint doi: https://doi.org/10.1101/2021.12.16.473086; this version posted January 7, 2022. The copyright holder for this preprint (which was not certified by peer review) is the author/funder, who has granted bioRxiv a license to display the preprint in perpetuity. It is made available under aCC-BY 4.0 International license.

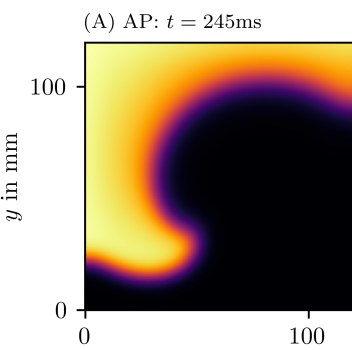

$x$ in $\mathrm{mm}$
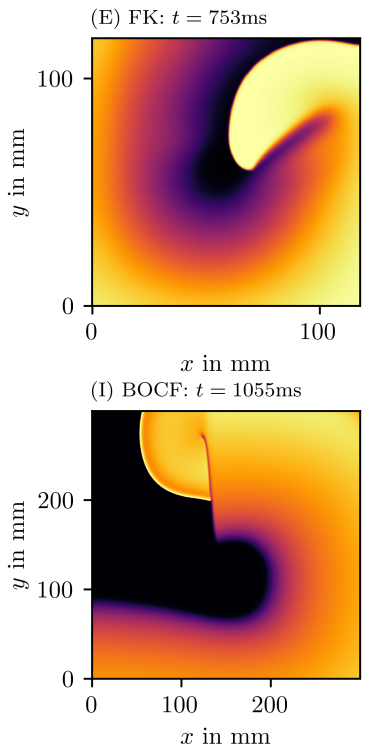

(M) OM: $t=12240 \mathrm{~m}$

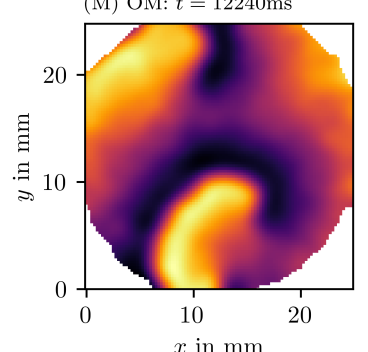

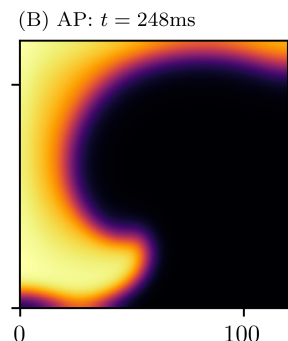

$x$ in $\mathrm{mm}$ (F) FK: $t=785 \mathrm{~ms}$
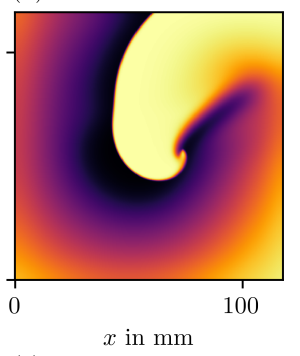

(J) BOCF: $t=1155 \mathrm{~ms}$

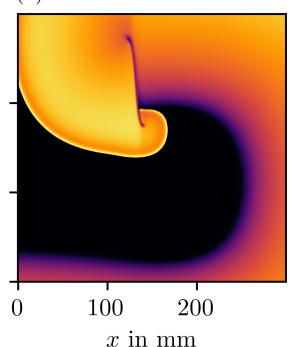

(N) OM: $t=12264 \mathrm{~ms}$

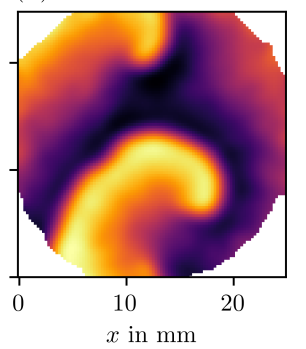

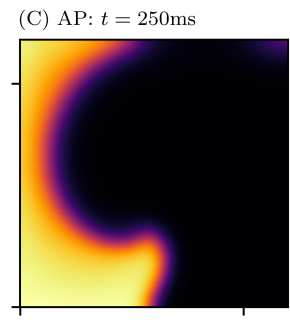

100

$x$ in $\mathrm{mm}$ (G) FK: $t=815 \mathrm{~ms}$

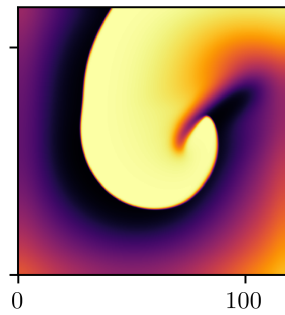

$x$ in $\mathrm{mm}$ (K) BOCF: $t=1250 \mathrm{~ms}$

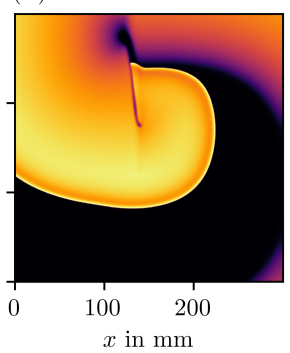

(O) OM: $t=12282 \mathrm{~ms}$

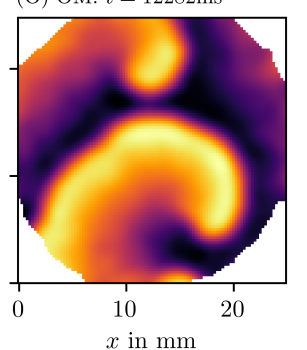

(C) AP: trajectory of PS

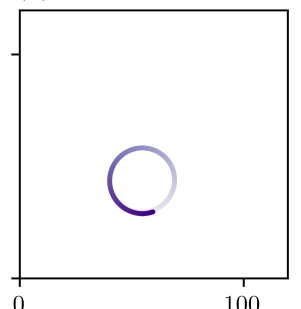

$x$ in $\mathrm{mm}$ (G) FK: trajectory of PS

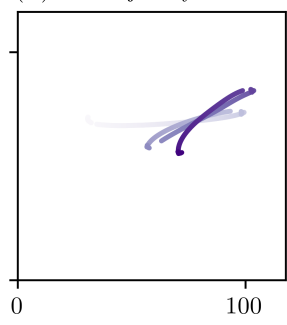

$x$ in $\mathrm{mm}$ (K) BOCF: trajectory of PS

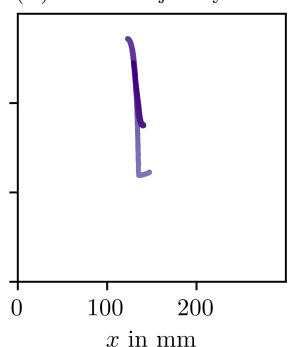

(O) OM: trajectory of PS

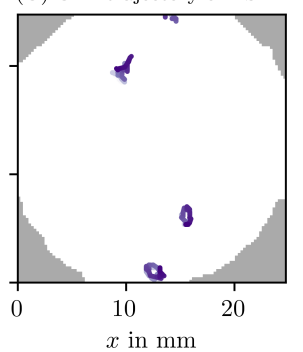

Fig 1. Qualitatively different tip trajectories of spiral waves in simulated cardiac tissue.

Rows show different reaction kinetics (AP, FK, BOCM) and an optical voltage mapping experiment on the last row. The first 3 columns show different snapshots in time and are colored according to local transmembrane voltage. Corresponding spiral wave tip trajectories are shown in the last column.

clinical data are only aiming to localize point singularities of phase.

The aim of this paper is to provide and compare several methods to numerically calculate the phase and the corresponding phase defects. We include a quantitative analysis of the results and the performance of the algorithms.

A summary of our workflow is presented in Fig 2 An image (e.g. of the transmembrane potential $u$ ) is in the first step converted into a phase $\phi$. Where jumps in phase are detected, the phase defect density $\rho$ (see below) will be much larger than zero. If 
desired, the field $\rho(\vec{r}, t)$ can be further processed to yield localised phase defect lines.
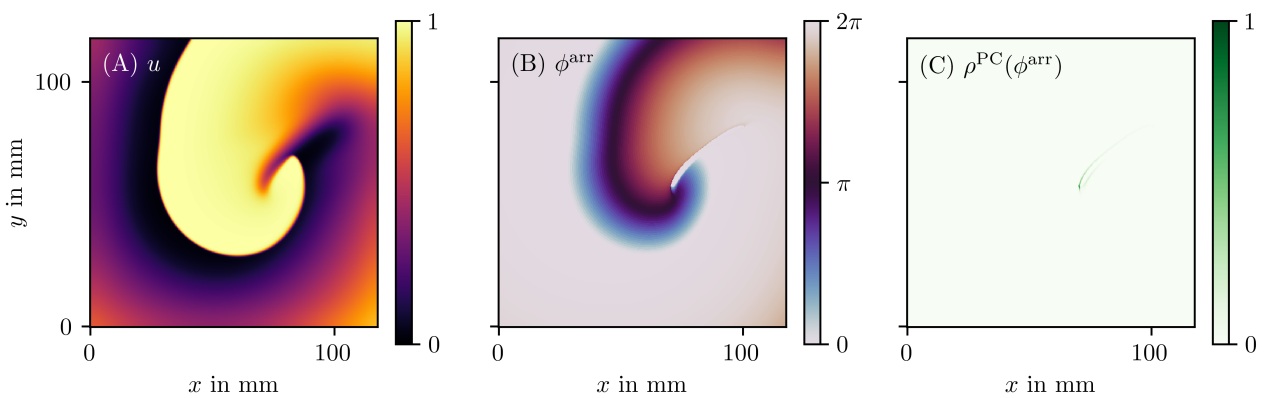

Fig 2. An example of the steps done in the process of constructing the phase defect field $\rho$.

(A) We start from the first phase state variable $u$, often the normalized transmembrane potential $V$. (B) Next, from this $u$ and possibly other state variables, the phase, here $\phi^{\text {arr }}$, is calculated. (C) Finally from the phase, the phase defect field is produced based on one of the PDL detection algorithms; here, the phase coherence (PC) method was used. These quantities are plotted over a $2 \mathrm{D}$ square domain of myocardial tissue in physical space. Colour is used to represent the values of the quantities mentioned in the top left corner. The same colouring will be used throughout this manuscript.

This manuscript is organized as follows: First, we briefly review the concepts of phase, phase singularities and phase defects (section 2). In the methods section 3 we outline our simulation methods and introduce several methods to trace phase defects in excitation patterns. Results of these methods and their performance are presented in section 4. We conclude this paper with a discussion and outlook (sections 5 and 6).

\section{Theoretical background}

\subsection{The concept of phase}

As remarked long ago by Winfree [14, many biological processes take values within a cycle rather than on the line of real numbers. Cardiac excitation is such an example, since during an action potential, the cell membranes depolarize and repolarize in normal circumstances along a predefined sequence, tracing out a closed loop in state space. To keep track of the relative state of cells along this cycle, the concept of phase can be used. In addition to the classical phase definition, called activation phase $\phi^{\text {act }}$ below, an alternative phase based on local activation times (LAT) was defined by Arno et al. 13. In this paper, a third phase $\phi^{\text {skew }}$ will be defined below as an approximation of $\phi^{\text {act }}$ when LAT are not available (Eq (7)).

Next, we will briefly review the previously defined phases $\phi^{\text {act }}$ and $\phi^{\text {arr }}$.

The activation phase $\phi^{\text {act }}$ is the phase as seen in a space spanned by two observables $V(\vec{x}, t)$ and $R(\vec{x}, t)$ in the system 2 6,10 . We henceforth assume that $V$ is representing the activation or depolarization of the medium, i.e. in cardiac context, we take $V$ to be the normalized transmembrane potential.

Even if there is only one variable $V$ observed, its time-delayed version 3], time derivative [5], or Hilbert transform [6] can be used as a linearly independent variable $R$. 
In numerical simulations, all state variables of the system can be observed, and any pair can be chosen as $(V, R)$. Then, one usually defines the activation phase as the polar angle of a state in the $(V, R)$-plane, relative to a reference point $\left(V_{*}, R_{*}\right)$ that lies within the cycle:

$$
\phi^{\text {act }}=\arctan 2\left(R-R_{*}, V-V_{*}\right)+c
$$

Here, the polar angle is returned by the two-argument inverse tangent: $\arctan 2(y, x)=\arctan (y / x)$ if $x \geq 0$ and $\arctan (y / x)+\pi \bmod 2 \pi$ if $x<0$. A constant $c$ can furthermore be added to make $\phi^{\text {act }}=0$ correspond to the resting state. The left column of Fig 3 visualises $\phi^{\text {act }}$ for the four data sets introduced in Fig 1 .

We recently proposed a second definition of phase that is based on the local activation time of tissue [15. The LAT, which is commonly used clinically, is defined as the time $t^{\text {arrival }}$ when the tissue locally depolarizes, i.e. when the transmembrane voltage $V$ at that point exceeds a value $V_{*}$. The LAT relative to the current time is the elapsed time:

$$
t^{\text {elapsed }}=t-t^{\text {arrival }}
$$

On other words, it is the time since the start of the last local activation. The arrival time phase $\phi^{\text {arr }}$ is just a mapping of $t^{\text {elapsed }}$ onto the interval $[0,2 \pi)$ by applying a sigmoidal function:

$$
\phi^{\mathrm{arr}}=2 \pi \tanh \left(t^{\mathrm{elapsed}} / \tau\right)
$$

where $\tau$ is the characteristic time of the cyclic process. Here we take $\tau$ equal to half of the action potential duration (APD) in the medium. Note that another sigmoid function instead of tanh could also have been chosen in Eq (3). The right column of Fig 3 visualises $\phi^{\text {arr }}$ for the same four data sets.

An asset of $\phi^{\text {arr }}$ is that the curves of equal $\phi^{\text {arr }}$ are precisely the isochrones that cardiologists work with during endocardial catheter mapping. Also, if $\tau$ is chosen large enough, repolarized (recovered) tissue will have $\phi^{\text {arr }} \approx 2 \pi$, such that this phase is not changing abruptly at the wave front and wave back, in contrast to $\phi^{\text {act }}$. A disadvantage of $\phi^{\text {arr }}$ is that it requires a dense temporal sampling (either in simulation or experiment), since otherwise staircase artefacts emerge. As a last remark, care should be taken when initializing $\phi^{\text {arr }}$ at the start of the observation window: If the previous LAT are unknown, so is $\phi^{\text {arr }}$ until a propagating wave has swept through the medium.

\subsection{Phase singularities and phase defects}

The analysis of spatial distributions of phase makes use of several concepts of complex numbers and complex analysis [16], such as contours, phase singularities and branch cuts. We now briefly review these concepts in the the context of cardiac excitation.

The detection of rotor cores from a spatial map of phase can be performed by calculating the total phase difference along a closed loop (contour) $C$ in the medium, usually taken on the cardiac surface:

$$
\Delta \phi=\oint_{C} d \phi
$$

Since the first and last point have the same phase, the resulting phase difference $\Delta \phi$ will return an integer multiple of $2 \pi$. Hence, one defines the topological charge 

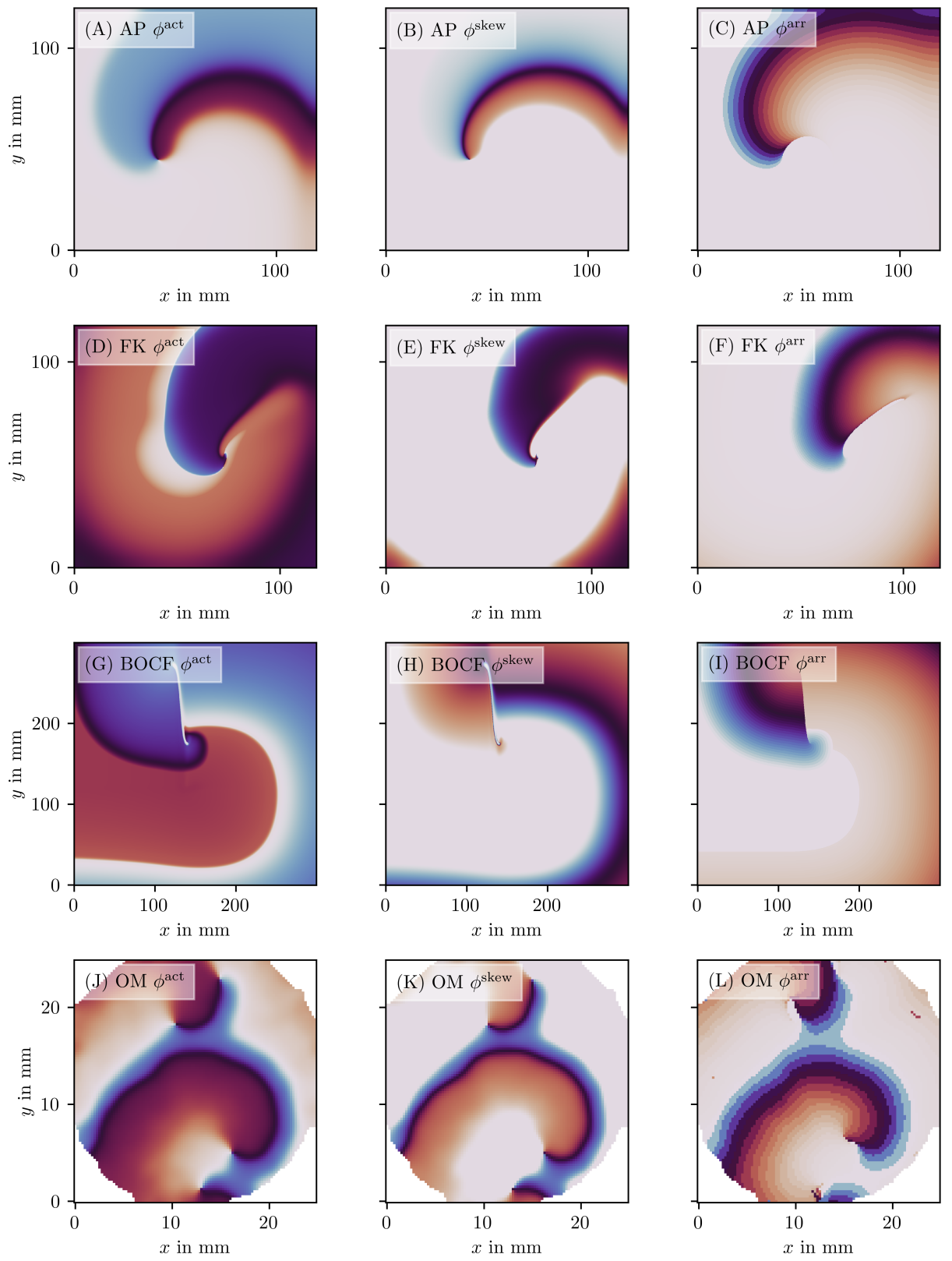

Fig 3. Illustration of the three different phases for one frame of four data sets.

The colour code represents the phase in modelled myocardial tissue in a 2D square domain in the same scale as in Fig $2 \mathrm{~B}$.

circumscribed by the contour as:

$$
Q=\frac{\Delta \phi}{2 \pi}=\oint_{\mathcal{C}} \frac{d \phi}{2 \pi}
$$

Now, in the classical theory [3, one assumes that the phase function $\phi(\vec{x})$ at a given time $t$ is continuous nearly everywhere, except in a few points where the phase is 
undefined. When using $\phi^{\text {act }}$, it can be seen that these points will correspond to $V=V_{*}, R=R_{*}$. In the immediate vicinity of such points, all phases are present (both in the $(V, R)$ plane and in the spatial phase map), hence this point is called a phase singularity (PS). Note that the existence of a phase singularity, where all different phases touch, within the contour region $\mathcal{C}$ is implied by the assumption of the original theory that the phase is a continuous function except at the PS.

In the generalized theory 12, 15], however, the phase is allowed to be discontinuous near a conduction block line, which also happens in the core of a linear-core spiral. Then, the contour $C$ cannot be shrunk to surround a single point, without having the contour cross an interface where $\phi$ changes abruptly. We call such transition zones phase defects (PD): phase defect lines (PDL) in 2D and phase defect surfaces (PDS) in 3D. The discontinuous behaviour of phase is more easily noted with $\phi^{\text {arr }}$ than with $\phi^{\text {act }}$, since $\phi^{\text {act }}$ also shows strong gradients near the wave front and wave back 15. As a result, we are convinced that PS detection algorithms that assume a continuous phase distribution are behaving non-robustly near such spatial phase transition, which motivates this work. Here, we will provide PD detection methods that are explicitly discriminating the PD structures, either as a sharp line, or in a probabilistic manner using PD density.

\section{Methods}

\subsection{Data generation and collection}

\subsubsection{Numerical methods for pattern generation}

The methods for PD detection developed here are designed to operate on excitation patterns, regardless of their generation. However, we here test the methods on numerical simulations in a cardiac monodomain setting. That is, in a rectangular Cartesian grid, we modeled forward evolution of a column matrix of state variables $\mathbf{u}(\vec{x}, t)$ according to a reaction-diffusion equation [17]:

$$
\partial_{t} \mathbf{u}=\mathbf{P} \Delta \mathbf{u}+\mathbf{F}(\mathbf{u})
$$

Here, the first component $u_{1}$ equals the normalized transmembrane potential $V$, and $\mathbf{P}=\operatorname{diag}(1,0, \ldots, 0)$ in order to enable wave propagation by diffusion of $V$. The number of state variables in $\mathbf{u}$ varies between the different mathematical models of cardiac myocytes, which are encoded in non-linear reaction functions $\mathbf{F}(\mathbf{u})$. To assess the reliability of our methods, we tried several reaction kinetics: Linear cores are known to occur with the Bueno-Orovio-Cherry-Fenton (BOCF) model for human ventricles [18] with parameter set 'PB' mimicking Priebe and Beuckelmann kinetics [19], the Fenton-Karma (FK) model [5] with either guinea pig (GP) or modified Luo-Rudy I (MLR-I) parameters. In addition, we investigated how the methods perform when operating on a circular spiral wave core by applying them to the Aliev-Panfilov (AP) model 20].

All simulations were executed on a 2D isotropic square domain of myocardial tissue using Neumann boundary conditions and a 5-point stencil for the Laplacian. Integrating in time is done using forward Euler stepping, with values per model given in Table 1 .

The spiral wave was generated by applying a S1S2 protocol: One side of the square is initially excited to generate a plane wave; if the central point of the medium has 
Table 1. Overview of the performed simulations with relevant parameters.

\begin{tabular}{ll|l|l|l|}
\hline & AP model & BOCF model & 18 & FK model \\
\hline grid size & $400 \times 400$ & $1200 \times 1200$ & $450 \times 450$ \\
$\Delta x$ & 1 & $0.25 \mathrm{~mm}$ & $0.262 \mathrm{~mm}$ \\
$\Delta t$ in ms & 0.01 & $0.009 \mathrm{~ms}$ & $0.163 \mathrm{~ms}$ \\
parameter set & default & PB & MLR-I \\
duration & 437.9 & $1250.01 \mathrm{~ms}$ & $815.31 \mathrm{~ms}$ \\
APD & 10.0 & $400.0 \mathrm{~ms}$ & $128.0 \mathrm{~ms}$ \\
$u$ for $\phi^{\text {act }}$ & $u$ & $s$ & $u$ \\
$v$ for $\phi^{\text {act }}$ & $v$ & $w$ & $1-v$ \\
$u_{\text {iso }}$ & 0.5 & 0.3 & 0.65 \\
$v_{\text {iso }}$ & 1.0 & 0.6 & 0.8 \\
$\phi_{0} /(2 \pi)$ & 0.15 & 0.0 & 0.35 \\
$\phi_{1} /(2 \pi)$ & 0.7 & 0.4 & 0.55 \\
\hline
\end{tabular}

finished repolarization, one quarter of the domain behind the traveling wave is stimulated, such that a spiral wave (cardiac rotor) is formed.

Three frames of the first model variable $u$ for each of the three chosen models resulting from numerical simulation were displayed in Fig 1 A-C.

\subsubsection{Optical voltage mapping experiments}

To test our methods on actual measurements, we used optical voltage mapping data derived from $10 \mathrm{~cm}^{2}$ monolayers of conditionally immortalized human atrial myocytes (hiAMs) following cardiomyogenic differentiation of these cells 21].

A voltage-sensitive dye is added to the culture after which a real-time recording can be made of the intensity of emitted light, which is a measure of the local transmembrane potential. For the used recordings, pixel size was $0.25 \mathrm{~mm}$ and the sampling time between frames was $6 \mathrm{~ms}$. Gaussian smoothing with a kernel size of three grid points has been applied to each frame. The data have been rescaled such that each grid point has unit variance in time. Then, arbitrary units have been defined such that the resting state corresponds to optical activity $u$ equal to zero, and the excited state to $u=1$. Three frames of one of these recordings can be seen in Fig 1 $1 \mathrm{D}$.

\subsection{A third phase definition}

The aforementioned phase definitions $\phi^{\text {act }}$ and $\phi^{\text {arr }}$ have each their downsides: Gradients in $\phi^{\text {act }}$ not only show PD but also wave fronts, and $\phi^{\text {arr }}$ requires intense sampling over time of the medium. We propose to combine the advantages of $\phi^{\text {act }}$ and $\phi^{\text {arr }}$ in a new phase, $\phi^{\text {skew }}$. This phase is designed as a computationally cheap approximation of the elapsed time phase $\phi^{\text {arr }}$ that can be calculated using $\phi^{\text {act }}$. By construction, it does not require the whole history of $V$, but can instead be calculated using $(V, R)$ at one point in time. In essence, $\phi^{\text {skew }}$ is a re-parameterization of $\phi^{\text {act }}$ :

$$
\phi^{\text {skew }}=h\left(\phi^{\text {act }}\right), \quad h(0)=0, \quad h(2 \pi)=2 \pi
$$

where $h$ is furthermore monotonically rising. Essentially, the cycle visited by cells during the action potential is now labeled in a more free manner than the classical polar 
coordinates in the $(V, R)$-plane. As can be seen in Fig 4 by plotting $\phi^{\text {act }}$ vs. $\phi^{\text {arr }}, \phi^{\text {arr }}$ is also a re-parameterization of $\phi^{\text {skew }}$.
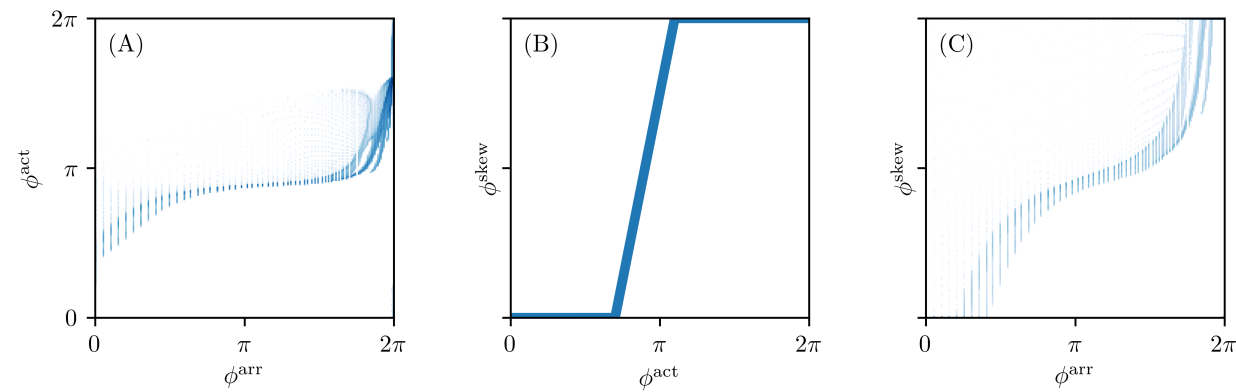

Fig 4. Correlation between the different phases, with data taken from the snapshot of the Fenton-Karma simulation in Fig 3.

Darker shading corresponds to higher logarithmic probability density.

In principle, one could fit a function $h$ to a plot of $\phi^{\text {arr }}$ vs. $\phi^{\text {act }}$, but we opt for a different approach different approach here and define a piece-wise linear function $h$ :

$$
h(\phi)= \begin{cases}0 & \phi \in\left[0, \phi_{0}\right], \\ 2 \pi \frac{\phi-\phi_{0}}{\phi_{1}-\phi_{0}} & \phi \in\left(\phi_{0}, \phi_{1}\right) \\ 2 \pi & \phi \in\left[\phi_{1}, 2 \pi\right] .\end{cases}
$$

Values for $\phi_{0}$ and $\phi_{1}$ were manually chosen for the different reaction kinetics models used, see section 2.1 and Table 1 .

Below, we will apply different phase defect detection methods on the three phases $\phi^{\text {act }}$, $\phi^{\text {arr }}$ and $\phi^{\text {skew }}$, to see which performs best in visualizing phase defect structures.

\subsection{Phase defect detection algorithms}

\subsubsection{Requirements for phase defect detection algorithms}

The aim of this paper is to provide and evaluate numerical methods that can be used as a successor of classical PS detection algorithms, but directed towards the detection of PDs instead. The following factors are taken into account when proposing the methods.

Zero or finite thickness. Due to the formation of a physical boundary layer (either by electrotonic effects or numerical smoothing, see 15]), a PD has a finite width in practice. Therefore we see two options. A first option is to see the PD as an idealized structure with zero thickness, situated near the steepest spatial variation of phase or a spatial discontinuity in the local activation time. A second option is to accommodate for the finite transition width, and describe the PD in a probabilistic manner, e.g. by regarding the phase gradient as a kind of PD density, below denoted as $\rho$. If desired, the PDL extent can then be determined by putting a threshold on $\rho$, a process which becomes easier if this density is normalized between 0 and 1 .

Vertex-based or edge-based detection. Since the algorithms work on data sets consisting out of phase values on a set of points, methods can be discriminated on whether they work on the nodes, edges or faces of the mesh. Since the PDs have co-dimension one, it 
is natural to consider them as being situated on edges of the computational grid, either in $2 \mathrm{D}$ or $3 \mathrm{D}$. However, the result of an edge-based method is not located on the original grid, such that methods that return values on the vertices of the grid (i.e. collocated with local phase data) can also be useful. Both edge-based and vertex-based methods are in contrast with PS detection: since PS have co-dimension 2, they are naturally calculated on the faces of the grid [5]22. Below we provide for most PD detection methods an edge-based and vertex-based variant. We currently test our methods on a 2D Cartesian grid only and leave the extension to 3D and irregular meshes to future work.

Taking phase differences. Since phase is a cyclic variable, phase differences should be taken with care. Spatial derivatives are implemented in such a manner that an integer multiple of $2 \pi$ is added in order to bring the result as closest as possible to zero. Also trigonometric functions are adjusted such that they are indifferent to $2 \pi$ differences. In some methods, the complex number $z=e^{\mathrm{i} \phi}$ is calculated and the absolute value $\|z\|$ is taken afterwards to obtain the phase instead of just using $\phi$. This will make sure that a large jump in phase is not just attributed to the phase being cyclic.

Performance. In the results section (section 4), the different algorithms are compared in computational speed and relative performance. Note that there is no ground truth in the detection of PDs, since the PD is effectively located where an algorithm finds it. Notwithstanding, to make a comparison between methods possible, we calculate the pixel-wise correlation between PDL points or densities returned by the different methods. If $\mathbf{A}$ and $\mathbf{B}$ are lists of the PDL densities returned by methods $\mathrm{A}$ and $\mathrm{B}$ in all $N$ points of the space-time grid, then we calculate from their means $\mu_{A}, \mu_{B}$ and standard deviations $\sigma_{A}, \sigma_{B}$ the covariance:

$$
\operatorname{Cov}(\mathbf{A}, \mathbf{B})=\frac{1}{N} \frac{\sum_{k=1}^{N}\left(A_{k}-\mu_{A}\right)\left(B_{k}-\mu_{B}\right)}{\sigma_{A} \sigma_{B}} .
$$

In the remainder of this section, nine different PD localization methods will be briefly discussed and/or introduced. For the vertex-based algorithms, the output is a discretized scalar field: a non-negative phase defect density $\rho(\vec{x}, t)$ at time $t$ that is defined in the points where phase is available to calculate the defect from (here either $\phi^{\text {act }}, \phi^{\text {arr }}$ or $\left.\phi^{\text {skew }}\right)$. For the edge-based algorithms, the output is a number $\sigma_{a b}$ computed from the pair of phases $\phi_{a}=\phi\left(\vec{x}_{a}\right), \phi_{b}=\phi\left(\vec{x}_{b}\right)$ found at the vertices connected by that edge, which could be regarded as making up a vector field.

\subsubsection{Interpolation between vertex-based and edge-based methods}

In what follows, $\vec{x}_{a}, \vec{x}_{b}, \ldots$ are positions of vertices $a$ and $b, \rho_{a}=\rho\left(\vec{x}_{a}\right), \phi_{a}=\phi\left(\vec{x}_{a}\right)$ and $\sigma_{a b}$ is a quantity defined on the edge of the mesh between $\vec{x}_{a}$ and $\vec{x}_{b}$. The set of vertices connected to vertex $a$ is $\mathcal{N}(a)$, containing $N_{a}$ elements. In a 3D Cartesian grid, $N_{a}=4$ inside the medium, but on the boundary of the domain or near obstacles, this value will be lower, but the following formulas can still be applied. See Fig 5 for graphical depiction of $\rho_{a}$ and $\sigma_{a b}$ on a portion of a Cartesian grid.

If a quantity arises naturally along an edge (e.g. a gradient), it can be interpolated onto the vertex grid using

$$
\rho_{a}=\frac{1}{N_{a}} \sum_{b \in \mathcal{N}(a)} \sigma_{a b}
$$




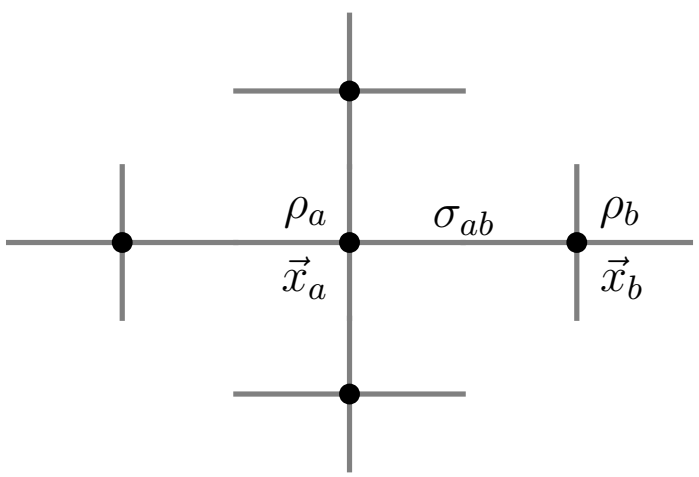

Fig 5. Overview of vertex- and edge-centered quantities.

For vertices $a$ and $b$ at positions $\vec{x}_{a}$ and $\vec{x}_{b}$, for which the phase $\phi$ has been calculated, we denote by $\rho_{a}$ the vertex-centered phase defect density, and $\rho_{b}$, respectively. For the edge $a \leftrightarrow b$ between the two vertices $a$ and $b$, we denote the edge-centered phase defect density by $\sigma_{a b}$. The vertices that $a$ is connected to are $a$ 's neighbourhood $\mathcal{N}(a)$.

Conversely, if a quantity is found at vertices, it can be allocated to the edges using linear interpolation:

$$
\sigma_{a b}=\frac{1}{2}\left(\rho_{a}+\rho_{b}\right)
$$

To distinguish between methods, the name of the method will be added in superscript, e.g. $\sigma^{\mathrm{CM}}, \rho^{\mathrm{PC}}$, etc.

\subsubsection{Overview of PD detection algorithms}

In the following, we will introduce several different methods to detect phase defects. A tabular overview of all the methods is given in Table 2

Table 2. Overview of existing and proposed algorithms for PD detection.

\begin{tabular}{|c|c|c|c|c|c|}
\hline abbr. & name & rationale & on vertices & on edges & calc. time \\
\hline $\mathrm{CM}$ & Cosine Method & $\begin{array}{l}\text { Jumps in phase mod } 2 \pi \text { are ok, inbe- } \\
\text { tween: PDL. }\end{array}$ & & $\checkmark$ & $3.74 \mathrm{~s}$ \\
\hline GLAT & $\begin{array}{l}\text { Gradient of Local Activa- } \\
\text { tion Time }\end{array}$ & LAT jumps at PDL and wave front. & & $\checkmark$ & $2.13 \mathrm{~s}$ \\
\hline RPG & Real Phase Gradient & Phase jumps at PDL. & & $\checkmark$ & $4.90 \mathrm{~s}$ \\
\hline CPG & Complex Phase Gradient & Phase jumps at PDL. & & $\checkmark$ & $13.31 \mathrm{~s}$ \\
\hline $\mathrm{PC}$ & Phase Coherence & Phase is not coherent at PDL. & & $\checkmark$ & $10.03 \mathrm{~s}$ \\
\hline $\mathrm{DM}$ & Dipole Moment & $\begin{array}{l}\text { Points on opposite side of PDL have } \\
\text { opposite "charge". }\end{array}$ & $\checkmark$ & & $13.74 \mathrm{~s}$ \\
\hline SVF & Spatial Vector Field & $\begin{array}{l}\text { Rotation of gradient is close to zero, ex- } \\
\text { cept at PDL. }\end{array}$ & $\checkmark$ & & $8.37 \mathrm{~s}$ \\
\hline $\mathrm{AM}$ & Angular Momentum & $\begin{array}{l}\text { Use the classical topological charge also } \\
\text { known as angular momentum. }\end{array}$ & $\checkmark$ & & $1.47 \mathrm{~s}$ \\
\hline IPM & Inflection Point Method & Change of sign of the phase Hessian. & & $\checkmark$ & $10.62 \mathrm{~s}$ \\
\hline
\end{tabular}

Calculation times are for 500 frames in a medium of $450 \times 450$ pixels on a Intel Core i7-10875H processor in a Numpy implementation without specific optimization for speed. 
Cosine method (CM). Tomii et al. 12 introduced the following quantity to visualize phase defects along an edge:

$$
\tilde{\sigma}_{a b}^{\mathrm{CM}}=\cos \left(\phi_{a}-\phi_{b}\right) .
$$

This method returns a value in $[-1,1]$, where low values indicate the presence of a phase defect.

To derive a normalized PD density with values in $[0,1]$, we modify this to:

$$
\sigma_{a b}^{\mathrm{CM}}=\frac{1}{2}\left[1-\cos \left(\phi_{a}-\phi_{b}\right)\right]
$$

and define $\rho_{a}^{\mathrm{CM}}$ via Eq 15 .

Gradient of local activation time (GLAT). It is expected that around a phase defect, the LAT does not vary smoothly but instead jumps across this line. This implies that the gradient in the neighbourhood of the PDL should be much larger than further away where neighbouring vertices are activated subsequently.

This relation is easily expressed using edges:

$$
\sigma_{a b}^{\mathrm{GLAT}}=t^{\mathrm{arrival}}\left(\vec{x}_{a}\right)-t^{\text {arrival }}\left(\vec{x}_{b}\right)
$$

Note that we are using a second condition here: If the elapsed time since excitation of either vertices is exactly zero, we still set $\sigma_{a b}^{\mathrm{GLAT}}$ to zero. The rationale here is that the jump is due to the wave front passing instead of pointing to a phase defect.

A vertex-based variant is found by averaging over all edges leaving the same vertex, see Eq (10), applied to the absolute value of the LAT difference:

$$
\rho_{a}^{\mathrm{GLAT}}=\frac{1}{N_{a}} \sum_{b \in \mathcal{N}(a)}\left|\sigma_{a b}^{G L A T}\right| .
$$

Real phase gradient (RPG). In previous work [13], we considered phase gradients, disregarding $2 \pi$ phase differences:

$$
\sigma_{a b}^{\mathrm{RPG}}=\left|\bmod \left(\phi_{a}-\phi_{b}+\pi, 2 \pi\right)-\pi\right|,
$$

returning values in $[-\pi, \pi]$. The normalized cosine method $(\mathrm{Eq}[13 \mathrm{p})$ can be seen as a mapping of this interval to a PD density taking values in $[0,1]$.

Complex phase gradient (CPG). The next method works in a similar fashion, but to avoid the modulo operation, we look for gradients in the complex number $z=\mathrm{e}^{\mathrm{i} \phi}$ :

$$
\sigma_{a b}^{\mathrm{CPG}}=\left|e^{i \phi_{a}}-e^{i \phi_{b}}\right| .
$$

From this a vertex-based density can be computed using Eq 10 .

Phase coherence (PC). Inspired by the literature of phase oscillators 23], we define the phase coherence of a vertex $a$ with neighbours $b$ as:

$$
p_{a}=\frac{1}{N_{a}}\left|\sum_{b \in \mathcal{N}(a)} e^{i \phi_{b}}\right| .
$$


The phase defect density is then defined as

$$
\rho_{a}^{\mathrm{PC}}=1-p_{a},
$$

such that $\rho$ is large when the coherence is low, since a low phase coherence is expected near a PDL. This index is normalized in $[0,1]$ with large values indicating high PD probability.

Note that applying the PC method to only two vertices connected via an edge delivers

$$
\sigma_{a b}^{\mathrm{PC}}=\frac{1}{2}\left|e^{i \phi_{a}}+e^{i \phi_{b}}\right|
$$

returning $\sqrt{1+\tilde{\sigma}_{a b}^{C M}}$.

Dipole moment (DM). Along a phase defect, the phase values that surround a given point are expected to be divided into two groups, one on either side of the PDL. We could perhaps detect this splitting by using the concept of the dipole moment of a charge distribution, where the complex number $z=\mathrm{e}^{\mathrm{i} \phi}$ takes the role of charge:

$$
\vec{p}\left(\vec{x}_{a}\right)=\sum_{\vec{x}_{b} \in \mathcal{N}(a)}\left[\vec{x}_{b}-\vec{x}_{a}\right] e^{i \phi_{b}} .
$$

The point-based phase defect field is then found by taking the norm of this complex vector:

$$
\rho^{\mathrm{DM}}=|| \vec{p} \|=\sqrt{\vec{p}^{*} \cdot \vec{p}} .
$$

where ${ }^{*}$ denotes complex conjugation.

When two vertices are connected by one edge, the edge-based implementation will recreate the CPG method. For this reason, no direct implementation of the latter was done. Still, edge-based values can be computed via interpolation, see Eq (11).

Spatial vector field (SVF). When Stokes' law is applied to the expression of topological charge, one finds

$$
Q=\int_{C} \vec{\nabla} \phi \cdot \overrightarrow{d \ell}=\iint_{S} \vec{\nabla} \times \vec{\nabla} \phi \cdot \overrightarrow{d S}
$$

where $C$ is the boundary curve to the region $S$. Since for a continuous field $\phi$, the rotation of the gradient $\vec{\nabla} \times \vec{\nabla} \phi$ vanishes everywhere, a continuous phase field cannot bear non-zero topological charge. Nevertheless, computing $Q$ for all faces of the grid has been used to find PSs 3 .

Inspired by the right-hand side of Eq $\sqrt{23}$, and replacing $\phi$ by $e^{i \phi}$ to get easier differentiation, we propose:

$$
\rho^{\mathrm{SVF}}=\|\vec{\nabla} \times \vec{\nabla} z\|
$$

The motivation for this method is that a phase defect is essentially a discontinuity in the field $\phi$. At such a discontinuity the rotation of the gradient may be different from zero. In our current implementation, we calculate the gradient in a vertex-based manner, e.g. $\partial_{x} u(x, y)=[u(x+d x, y)-u(x-d x, y)] /(2 d x)$, such that the result is also vertex-based. 
Angular momentum (AM). A classical method to detect the central region in a spiral wave is using the pseudo-vector: 24

$$
\vec{L}=\vec{\nabla} u \times \vec{\nabla} v .
$$

Far away from the spiral core, the activation resembles a plane wave, making $\vec{\nabla} u$ nearly parallel to $\vec{\nabla} v$, such that $\vec{L} \approx \overrightarrow{0}$ except near the core of the spiral. Since PS can be seen as a limit of a PDL with vanishing length, we will visualize

$$
\rho^{\mathrm{AM}}=\|\vec{L}\| .
$$

An edge-based method can be derived by taking:

$$
\sigma_{a b}^{\mathrm{AM}}=\left(\rho_{a}^{\mathrm{AM}}+\rho_{b}^{\mathrm{AM}}\right) / 2 .
$$

Inflection point method (IPM). Given that a PDL in practice connects two regions of different phase in an abrupt but continuous manner, it is interesting to look where the phase transition is the steepest, and localize the phase defect there.

For 1D functions, an inflection point is found where $f^{\prime}(x)$ changes sign. This can be translated to the condition $f^{\prime \prime}(x)=0$. To find the same region for a $2 \mathrm{D}$ function, we express that we want an inflection point when stepping in the direction of the local phase gradient. With $\vec{e}_{g}$ as the normalized gradient vector:

$$
\vec{\nabla} \phi=\vec{g}=g \vec{e}_{g},
$$

the spatial derivative in the gradient direction is $\partial_{g}=\vec{e}_{g} \cdot \vec{\nabla}$. With this, $\partial_{g} \phi=g$, and the concavity in the direction of the gradient becomes:

$$
F(\vec{x})=\sum_{i, j} g^{i} g^{j} \partial_{i j}^{2} \phi=\sum_{i, j} g^{i} g^{j} H_{i j},
$$

where the Hessian of the phase is $H_{i j}=\partial_{i j}^{2} \phi$. Hence, the PD can be found as the set of points where $F(\vec{x})=0$. This method is unlike the mentioned algorithms above, since it immediately returns a line, i.e. PDL of zero thickness. Note that in practice, one needs to impose a minimal value of $\left\|\rho^{G L A T}\right\|$ such that the background region with low PDL density is filtered out.

To compare this method to the other algorithms, we color the edges where $F(\vec{x})$ changes sign with the phase gradient along that edge, i.e.

$$
\sigma_{a b}=\sigma_{a b}^{R P G} H\left[-F\left(\vec{x}_{a}\right) F\left(\vec{x}_{b}\right)\right]
$$

with the Heaviside function $H$.

\subsection{Visual representation of the methods}

For the methods that return a vertex-based PD density $\rho$, we simply color the pixels in the rectangular grid according to $\rho$. For the methods that return an edge-based PD indicator $\sigma$, we color the dual grid, i.e. we color every point in the plane according to its nearest edge. This results in a coloring of the plane using pixels that are $45^{\circ}$ tilted and centered around the midpoint of edges in the original grid. In this way, interpolation between edges and vertices does not affect the presented results. 


\subsection{Post-processing of phase defects}

Having obtained a PD density $\rho(\vec{x})$ using one of the methods, we keep only points above a threshold value $\rho_{c}$ to obtain a set of points on the PDL, and connect it using the minimal spanning tree graph algorithm. Thereafter, the smallest branches of each tree are cut to gain a discrete representation of a phase defect line, centered at the vertices of the image grid.

To measure PDL length $L$, the PDL points are connected by line segments; the sum of their lengths is taken as an estimate to the PDL length.

To measure PDL precession speed, we first selected a spatial region where only one PDL was seen during the timespan of interest. Then, we performed principal component analysis (PCA) to the point cloud of the PDL at all time instances to obtain the main vector of alignment $\vec{e}_{1}$. The angle between this vector and the positive $\mathrm{X}$-axis is taken to be $\beta$, after which linear regression of $\beta(t)=\beta(0)+\omega t$ yields an estimate for the precession frequency $\omega$ and the precession period $T=\frac{2 \pi}{\omega}$.

\section{Results}

We here apply the different proposed detection methods for the three phases $\phi^{\text {act }}, \phi^{\text {arr }}$, and $\phi^{\text {skew }}$. We do this in three cardiac monodomain models and compare performance of the methods (section 3.1.1). Finally, we apply a selection of methods to an experimental dataset obtained by optical voltage mapping of a monolayer culture of cardiac cells (section 3.1.2.

\subsection{Comparison of different phase definitions}

Fig 3 shows the three phase definitions applied to a snapshot of the three monodomain models and an optical voltage mapping experiment.

The AP model shown in the first row of Fig 3 produces a rigidly rotating spiral. With $\phi^{\text {act }}$ and $\phi^{\text {skew }}$, a PS is seen. However, due to the thresholding on $V$ used to determine LAT, the inner part of the core region is never excited, such that $\phi^{\text {arr }}$ shows an abrupt change at the trajectory of the classical PS, which will be picked up as a PD below.

In the simulations with linear core (FK and BOCF models), $\phi^{\text {act }}$ shows sudden transitions at the rotor core and the wave front, while $\phi^{\text {skew }}$ and $\phi^{\text {arr }}$ only show a distinct phase gradient near the conduction block line.

The optical voltage mapping experiment in Fig 3J-L shows apparent PSs for $\phi^{\text {act }}$ and $\phi^{\text {skew }}$, but an extended phase defect for $\phi^{\text {arr }}$. Hence, at first sight, it resembles the AP spiral, but this relation will be further investigated below using the PD detection techniques outlined above.

Fig 4 shows a scatter plot between the different phases for the FK frame shown in Fig 3. We took the convention that the phase at the resting state is 0 . The skewed phase $\phi^{\text {skew }}$ with parameters tuned as outlined above resembles the elapsed time phase $\phi^{\text {arr }}$ much closer than the state space phase $\phi^{\text {act }}$. In short, $\phi^{\text {skew }}$ is an approximation to $\phi^{\text {arr }}$ that 
does not require observation of the system during the previous excitation sequence.

\subsection{Comparison of phase defect detection methods in simulations}

\subsubsection{Spatial maps of PD density}

We have applied all phase defect detection methods (section 3.3 to all simulations, for all phases. For definiteness, we only show the result for the FK model in Fig 6 and 7 , but the others can be found in the Supplementary Materials (section 6, 6, 6, and 6).

In the following, we will present a selection of the results exhibiting the common features and our main observations regarding the different methods.

In general, all methods return low densities away from the wave front and PDL and higher values near the region of interest, although the precise PD density distribution is different between the methods.

In Fig 6B, the GLAT method clearly shows the conduction block line at the rotor core. Since LAT is discontinuous there, the set of points is thin such that small gaps can be seen. At the rightmost part, the PDL doubles, since the process of reaching and leaving the rightmost turning point both leave a discontinuity in LAT. Moreover, the PDL's precise location depends on the chosen threshold $V_{*}$.

The AM method (Fig 6C) locates only the site where the wave front ends on the PDL, and slightly stresses the wave front. This is consistent with this method traditionally being used for PS detection.

The other methods in Fig 607 are phase-based. In each case, the wave front is most clearly seen as an artefact using $\phi^{\text {act }}$, less visible using $\phi^{\text {skew }}$ and absent in $\phi^{\text {arr }}$.

The CM, RPG and CPG methods (Fig 6D-L) and PC and DM methods (Fig 74-F) give all qualitatively similar results: With $\phi^{\text {act }}$ and $\phi^{\text {arr }}$, not only the PDL but also the end point of the wave front (tip) is stressed. The $\phi^{\text {arr }}$-variant distinctly shows the PDL, as the wave front is filtered out by the definition of $\phi^{\text {arr }}$.

The IPM method shows a line that is only one pixel wide, as it was designed to localize the PD at the site of steepest phase variation.

Finally, the SVF method yields many points in the region of interest, but the result is noisy even in this idealized simulation.

\subsubsection{Correlation between the methods}

After interpolation to the same vertex-based grid, we computed the mutual correlation of the different methods using Eq (9), see Table 3. The methods CM and RPG are most strongly correlated, and AM is uncorrelated to the other methods, which can be expected as it highlights PSs rather than PDLs. The correlation information is graphically depicted in Fig 8 , where more similar methods are plotted closer to each other 25]. 

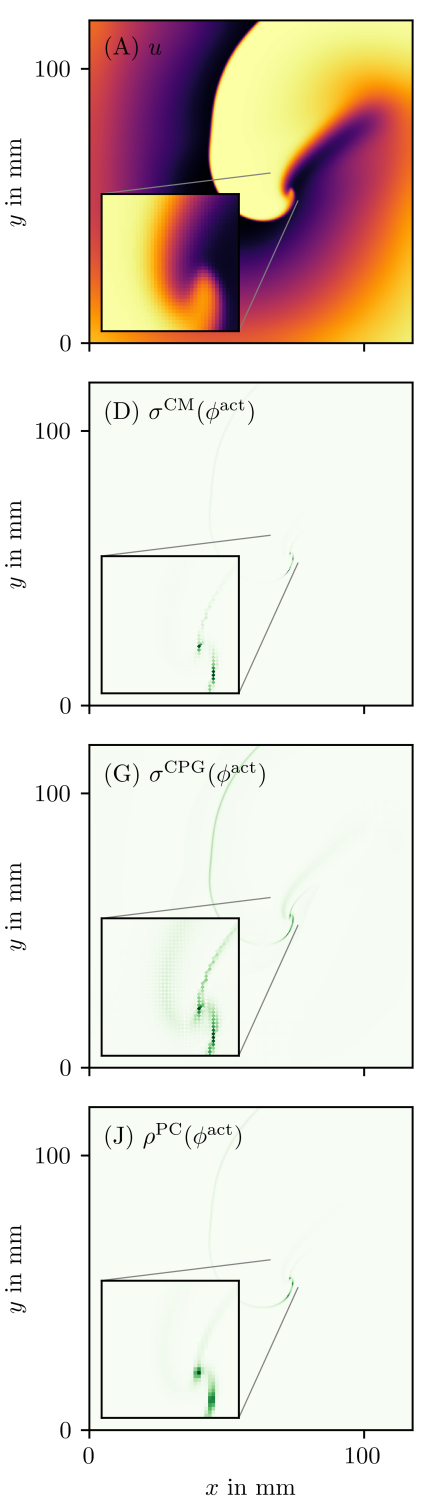
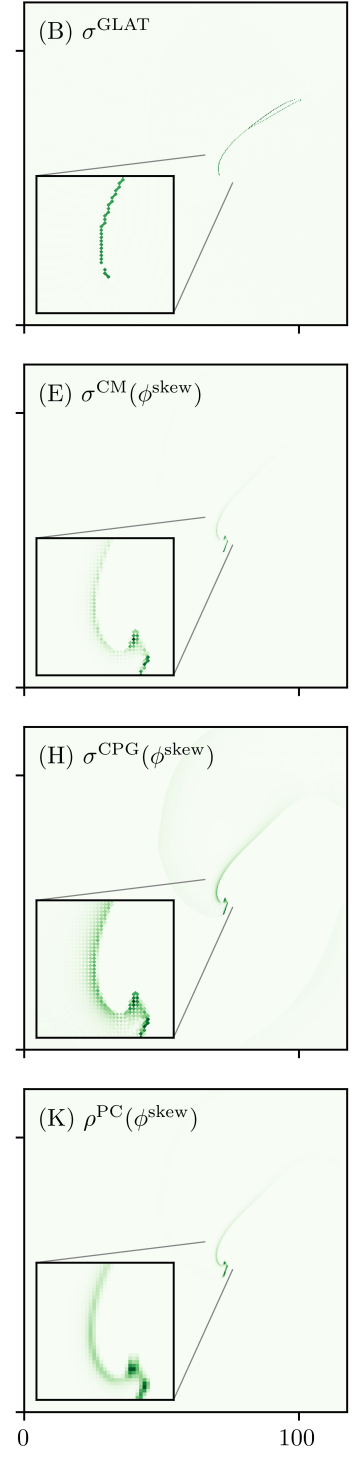

$x$ in $\mathrm{mm}$
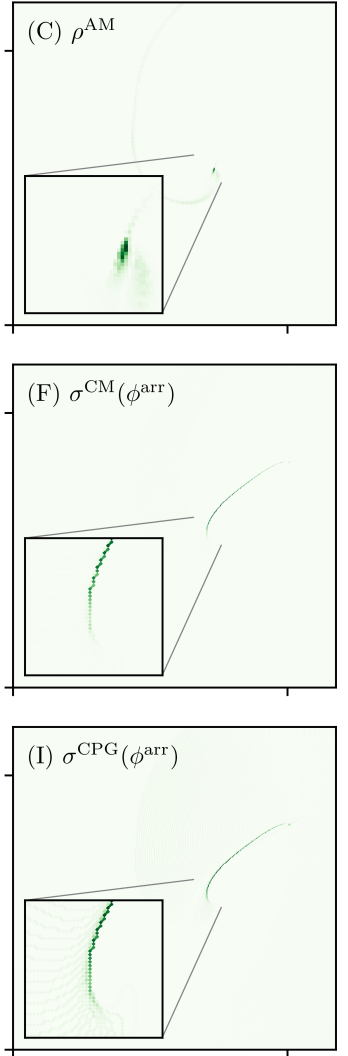

(L) $\rho^{\mathrm{PC}}\left(\phi^{\text {arr }}\right)$

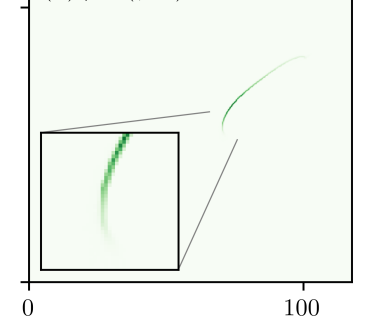

$x$ in $\mathrm{mm}$

Fig 6. Overview of phase defect detection methods for one snapshot of the FK data set.

The phase defect on vertices $\rho$ or edges $\sigma$ is measured in arbitrary units. The same colouring as in Fig $2 \mathrm{C}$ is used here. As the phase defect line has a width of only a few grid points, we zoom in around the turning point to get a better view of the structure on the grid. For reference, we also show the corresponding frame of the transmembrane voltage $V=u$ in panel a1.

\subsection{Comparison of phase defect detection methods in an optical voltage mapping experiment}

We also applied the different phase definitions and detection methods to the excitation sequence observed in a hiAM monolayer, as detailed in section 3.1.2. Fig 10A shows the optical intensity at a given time in a multiple-spiral state. The non-phase methods 

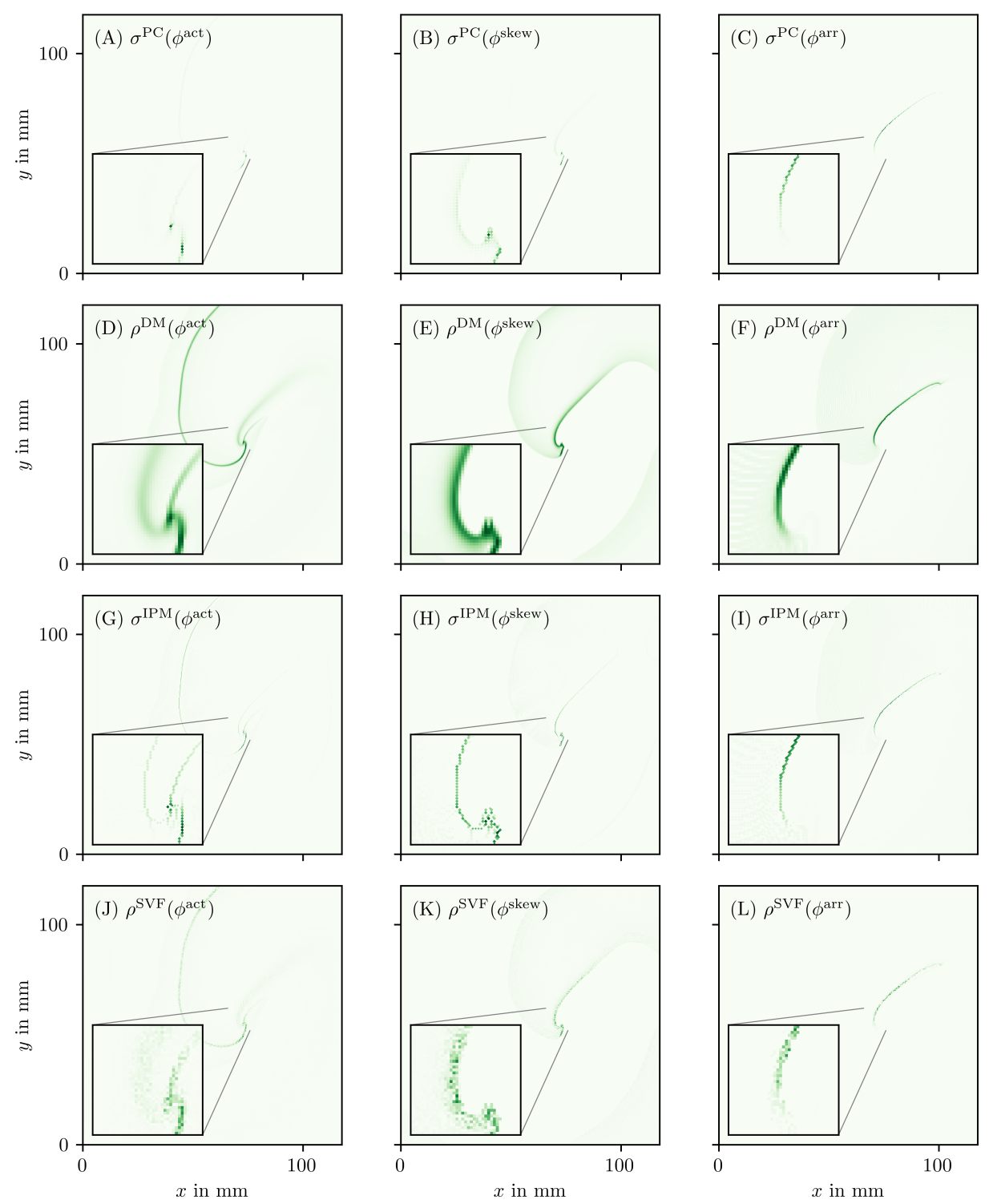

Fig 7. Overview of more phase defect detection methods for one snapshot of the FK data set as in Fig 6.

Each row shows a detection algorithm, applied to 3 different phase definitions.

GLAT and AM in Fig 10B-C show non-zero densities at several positions that are similar in both methods. The four most intense points, at which either a PS or PDL could be present, are confirmed by the other methods (CM, RPG, CPG, PC, DM, IPM) using $\phi^{\text {act }}$ and $\phi^{\text {skew }}$. When using $\phi^{\text {arr }}$, the same 4 points are prominent, but they extend to a line (PDL) since the LAT and $\phi^{\text {arr }}$ keep track of the recent history of excitation. Compared to the simulation data, more background structures are seen in the optical voltage mapping data, such as borders of excited regions and a staircase effect in LAT due to the time sampling.

Also for this data, we present the correlation in Table 4 and graphically in Fig 12 . 
bioRxiv preprint doi: https://doi.org/10.1101/2021.12.16.473086; this version posted January 7, 2022. The copyright holder for this preprint (which was not certified by peer review) is the author/funder, who has granted bioRxiv a license to display the preprint in perpetuity. It is made available under aCC-BY 4.0 International license.

Table 3. Correlations of vertex-based phase defects $\rho$ based on $\phi^{\text {arr }}$ for the

FK simulation.

\begin{tabular}{llllllllll}
\hline Method & AM & CM & CPG & DM & GLAT & IPM & PC & RPG & SVF \\
\hline AM & & 0.001 & 0.0023 & 0.00037 & -0.0026 & -0.0040 & 0.0013 & -0.0018 & 0.0077 \\
CM & 0.001 & & 0.40 & 0.63 & 0.51 & 0.63 & 0.89 & 0.85 & 0.68 \\
CPG & 0.0023 & 0.40 & & 0.76 & 0.27 & 0.79 & 0.53 & 0.48 & 0.51 \\
DM & 0.00037 & 0.63 & 0.76 & & 0.39 & 0.87 & 0.71 & 0.80 & 0.61 \\
GLAT & -0.0026 & 0.51 & 0.27 & 0.39 & & 0.42 & 0.44 & 0.49 & 0.39 \\
IPM & -0.0040 & 0.63 & 0.79 & 0.87 & 0.42 & & 0.67 & 0.8 & 0.54 \\
PC & 0.0013 & 0.89 & 0.53 & 0.71 & 0.44 & 0.67 & & 0.77 & 0.83 \\
RPG & -0.0018 & 0.85 & 0.48 & 0.80 & 0.49 & 0.8 & 0.77 & & 0.61 \\
SVF & 0.0077 & 0.68 & 0.51 & 0.61 & 0.39 & 0.54 & 0.83 & 0.61 & \\
\hline
\end{tabular}
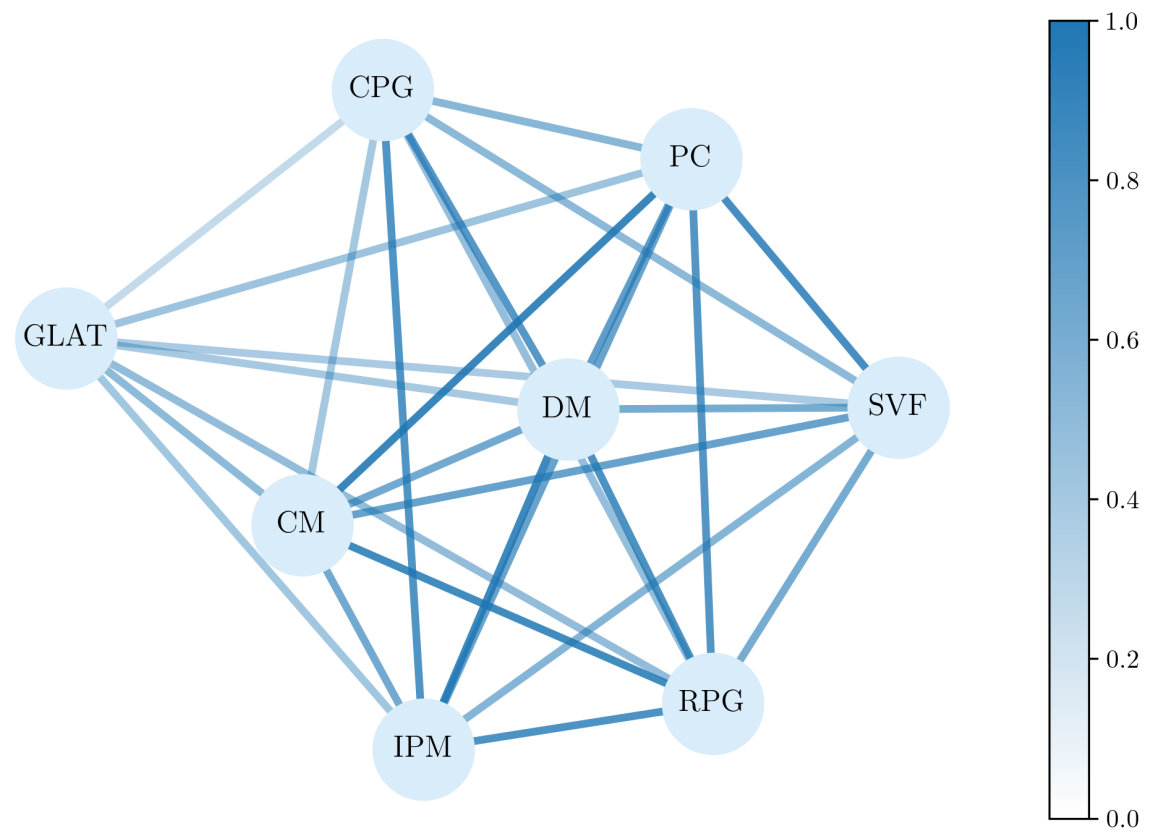

Fig 8. Correlations in Table 3 displayed as a weighted graph.

Visualisation using Networkx 25. Colour of edges represents the correlation between the two connected methods. Distance between nodes roughly represents how similar they are.

\subsection{Properties of PDLs in simulation and experiment}

The presented methods allow to characterize the observed PDLs in terms of length $L$ and orientation angle $\beta$, which is a further step in the quantitative analysis of excitation patterns.

Fig 13 shows the length over time of one PDL, in simulations and experiment. The PDL is detected using the arrival time phase $\phi^{\text {arr }}$ as input for the phase coherence method (PC). We observe that the PDL length varies over time. Its time-averaged value is summarized in Table 5 .

In both simulations and experiment, we also estimated the precession period $T$ of the 

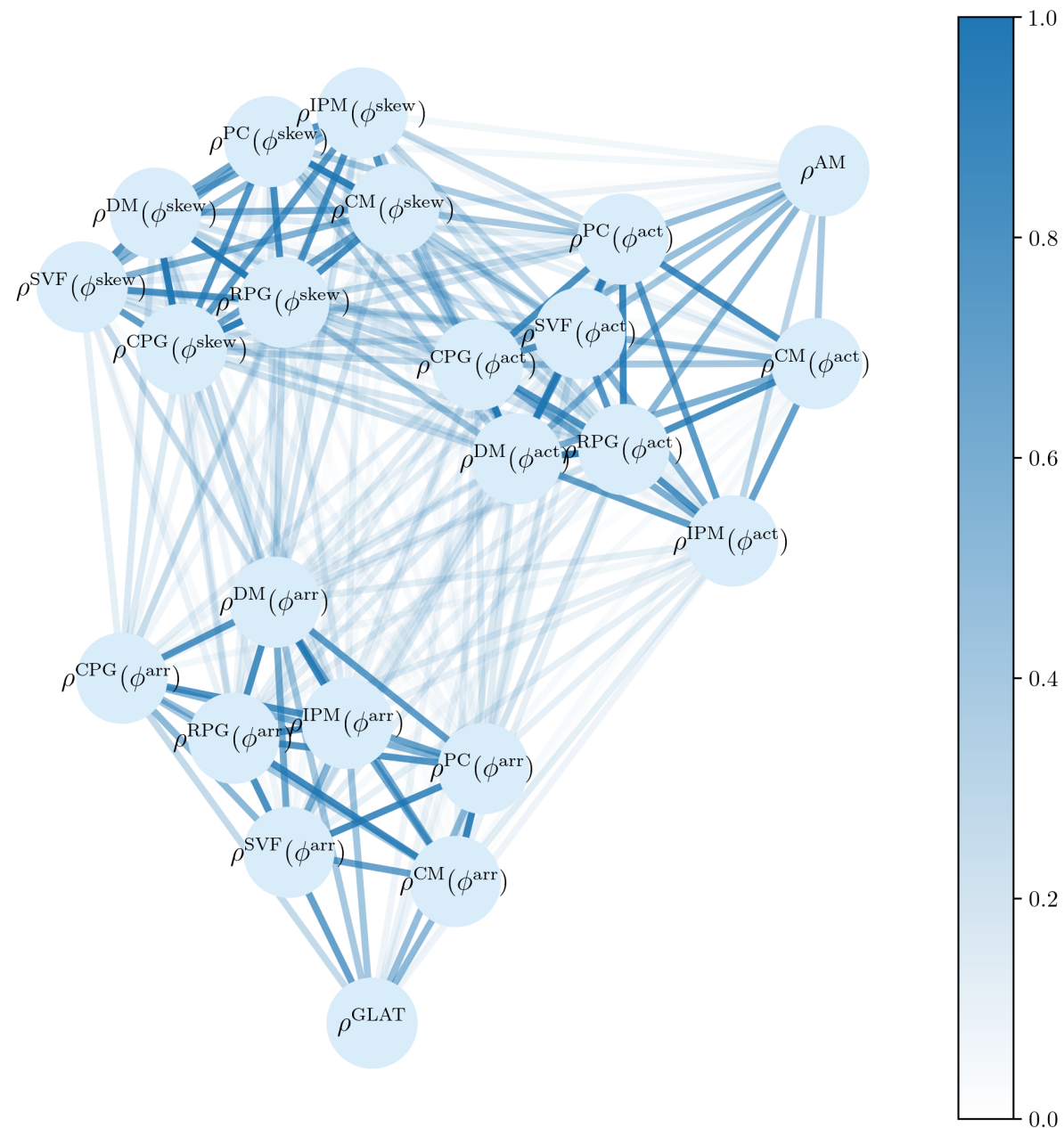

Fig 9. Correlations for all methods displayed as a weighted graph.

Data are visualised in the same way as in Fig 8 . We note that the different methods fall apart in clusters of the phase definition rather than the precise PD algorithm used.

Table 4. Correlations of vertex-based phase defects $\rho$ based on $\phi^{\text {arr }}$ for the OM data.

\begin{tabular}{llllllllll}
\hline Method & AM & CM & CPG & DM & GLAT & IPM & PC & RPG & SVF \\
\hline AM & & 0.13 & 0.19 & 0.27 & 0.13 & 0.15 & 0.23 & 0.15 & 0.19 \\
CM & 0.13 & & 0.31 & 0.58 & 0.039 & 0.58 & 0.7 & 0.94 & 0.20 \\
CPG & 0.19 & 0.31 & & 0.7 & 0.013 & 0.65 & 0.62 & 0.3 & 0.24 \\
DM & 0.27 & 0.58 & 0.7 & & 0.026 & 0.78 & 0.81 & 0.65 & 0.30 \\
GLAT & 0.13 & 0.039 & 0.013 & 0.026 & & -0.027 & 0.078 & 0.019 & 0.039 \\
IPM & 0.15 & 0.58 & 0.65 & 0.78 & -0.027 & & 0.67 & 0.65 & 0.26 \\
PC & 0.23 & 0.7 & 0.62 & 0.81 & 0.078 & 0.67 & & 0.64 & 0.39 \\
RPG & 0.15 & 0.94 & 0.3 & 0.65 & 0.019 & 0.65 & 0.64 & & 0.19 \\
SVF & 0.19 & 0.20 & 0.24 & 0.30 & 0.039 & 0.26 & 0.39 & 0.19 & \\
\hline
\end{tabular}

PDL, see Table 5 and Fig 14 . The orientation of the PDL changes almost linearly in all cases, hence we observe quite low variance along the fit linear functions. On the one hand, in the FK and BOCF simulations, we see that $\beta$ almost stays constant, but 

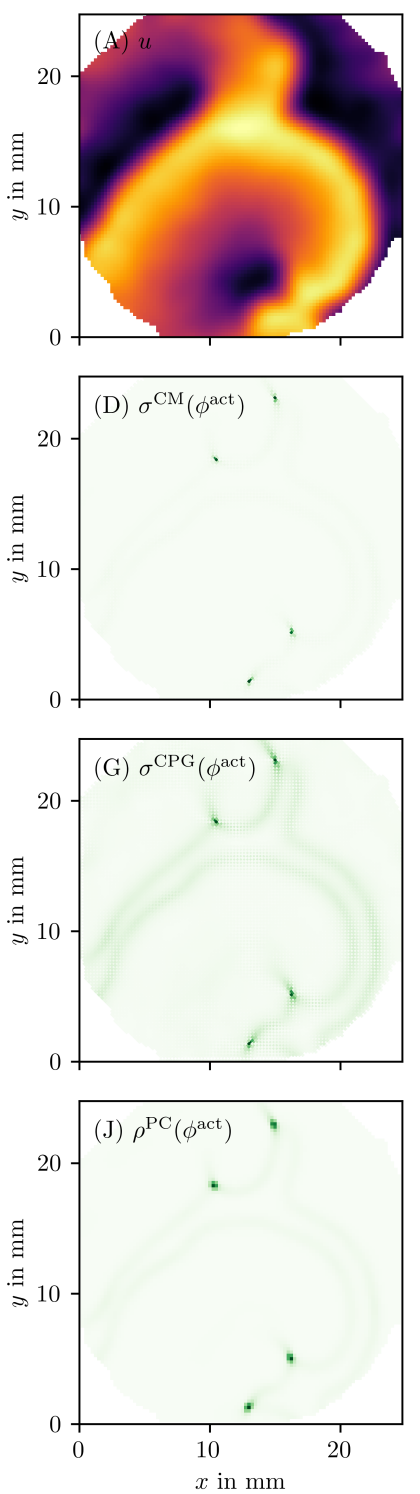
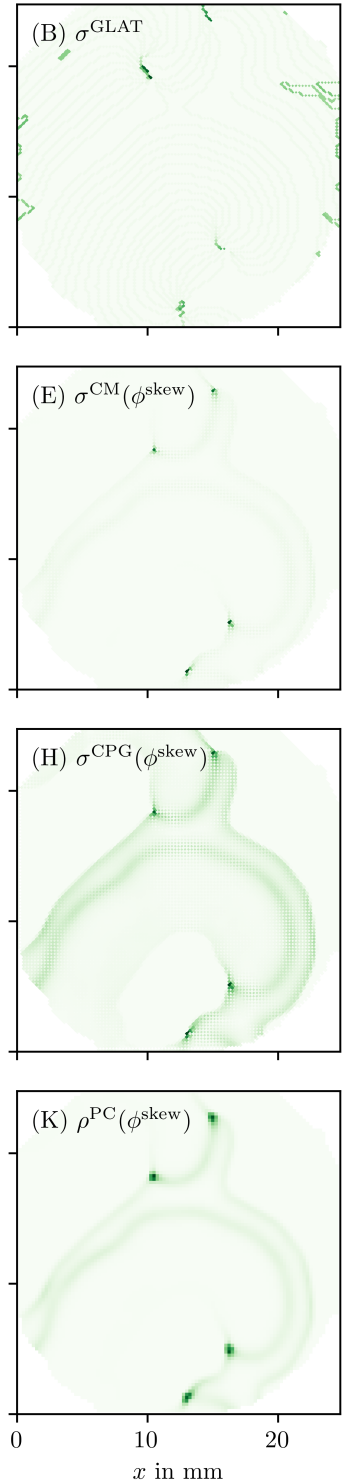
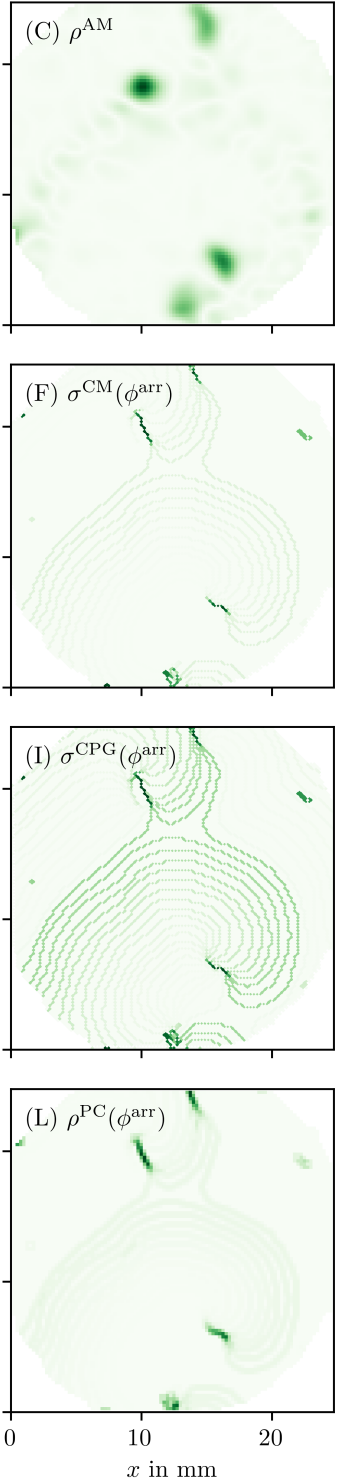

Fig 10. Overview of phase defect detection methods for one snapshot of the optical voltage mapping data.

The data are presented in the same way as in Fig 6

Table 5. Statistics of one PDL's length and precession over time.

\begin{tabular}{lrr}
\hline model / experiment & average length $L$ & precession period $T$ \\
\hline AP simulation & $(15.07 \pm 0.67)$ & $(33.123 \pm 0.011)$ \\
FK simulation & $(35.9 \pm 6.2) \mathrm{mm}$ & $(1223 \pm 29) \mathrm{ms}$ \\
BOCF simulation & $(76.1 \pm 7.8) \mathrm{mm}$ & $(57508 \pm 87) \mathrm{ms}$ \\
hiAM optical voltage mapping & $(4.01 \pm 0.55) \mathrm{mm}$ & $(169.2 \pm 9.3) \mathrm{ms}$ \\
\hline
\end{tabular}

We observe similar values for other PDLs in the data.

slightly precesses in one direction. On the other hand, in the AP model and optical voltage mapping data, the precession takes place in a much shorter period of time: $T$ is 

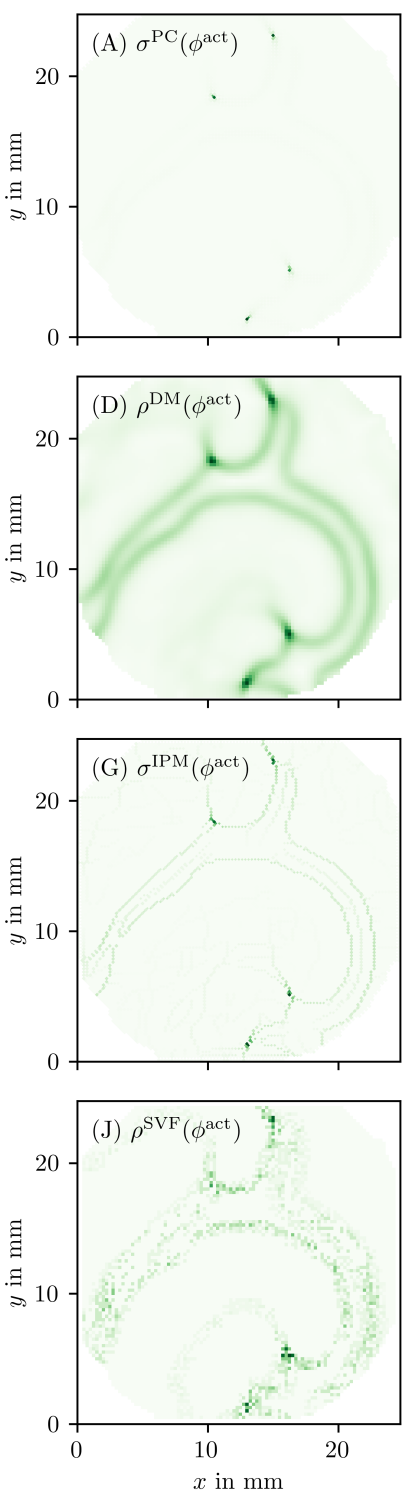
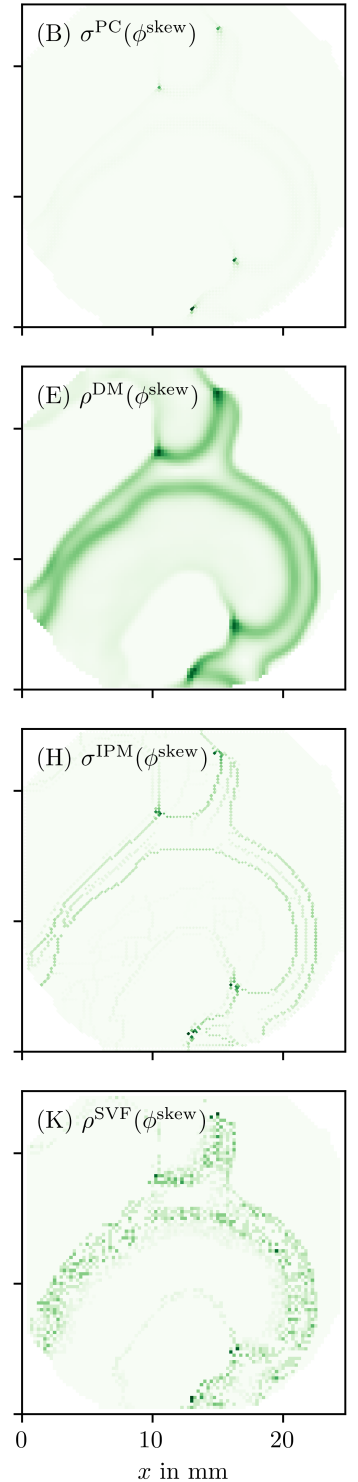

Fig 11. Overview of more phase defect detection methods for one snapshot of the optical voltage mapping data as in Fig 10 .

just 1.5 to 3 times longer than the APD in both of those cases.

\section{Discussion}

In this paper, we provide and compare several numerical methods to detect a phase defect line, a recently proposed structure present at the core of a rotor as an alternative for the classical phase singularities [12,13. Here, we attempt to improve the simple PDL detection methods from these works (CM \& RPG) and tested them on simulations and experimental data. 


\section{GLAT}

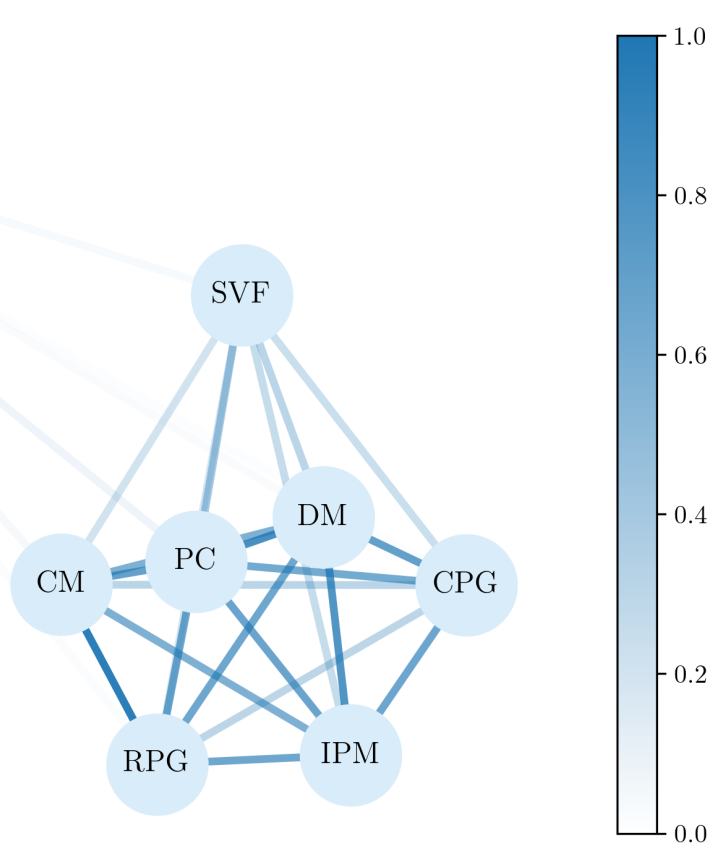

Fig 12. Correlations in Table 4 displayed as a weighted graph.

Data are visualised in the same way as in Fig 8 .
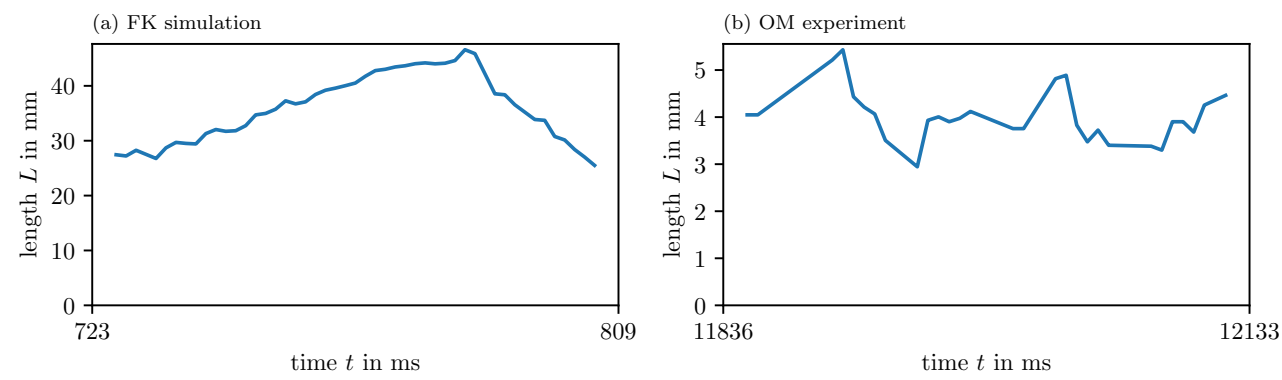

Fig 13. Length of detected PDLs over time.

For one of the PDLs detected by the PC method for $\phi^{\text {arr }}$, we show how its length changes over time. This length fluctuates around an average value.
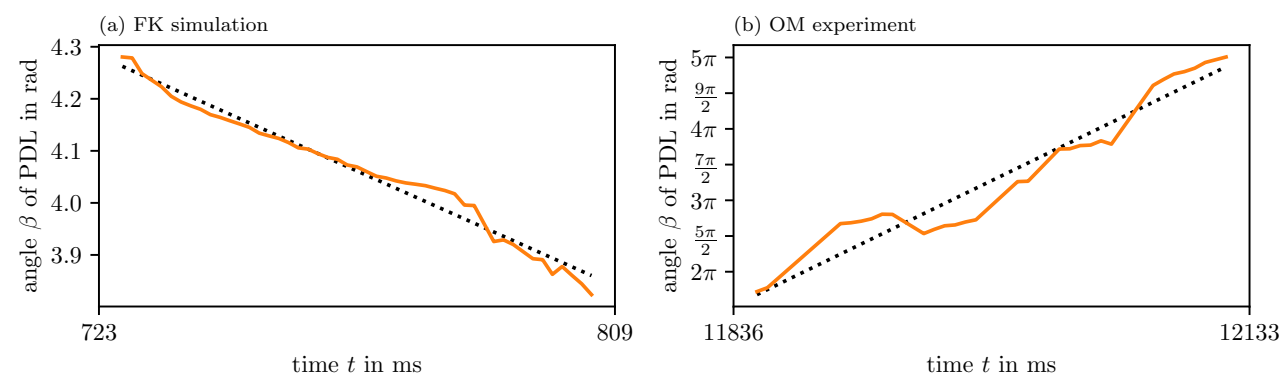

Fig 14. PDL orientation over time.

For these figures, we use the same PDLs as in Fig 13 and Table 5 . 
Several phase-based algorithms were applied, not only the 'classical phase' $\phi^{\text {act }}$ but also the recently introduced LAT-based phase $\phi^{\text {arr }}$ 13], since the LAT better keeps the spatio-temporal activation and therefore more clearly shows extended phase defects. In addition to a systematic comparison between detection methods, we also introduce a third phase, the 'skewed phase' $\phi^{\text {skew }}$ in this work. $\phi^{\text {skew }}$ was designed as a way to estimate $\phi^{\text {arr }}$ from a single snapshot. This is useful in the post-processing of data from experiments or simulations, where a sparse time-sampling was used. Although $\phi^{\text {skew }}$ filters the wave front and back better than $\phi^{\text {act }}$, we still find that direct measurements of LAT and $\phi^{\text {arr }}$ produce better-resolved PDLs (cf. Fig 6).

When comparing the different methods to convert phase into a phase defect, we find a good performance and strong correlation between the previously coined cosine method $[12$ and the real phase gradient method [13]. Both methods are based on the same idea (measuring angular differences along a circle) and therefore the correlation comes as no surprise. Qualitatively similar performance is found by related methods (CPG, CM, PC). The IPM method also works well. Some advanced methods such as DM, and SVF actually performed worse in terms of contrast and noise suppression. Of special interest are the classical AM method (AM) 24], which consistently finds the end point of the wave front, even if lying on a PDL, and the GLAT method. The gradient of LAT correctly identifies the PDL and locates it very sharply (by construction); however, its precise location depends on the chosen threshold $\left(V_{*}\right)$ to classify tissue as excited or not. Regarding the calculation time, we find that the CM, AM and GLAT are the fastest and therefore recommended to use for processing larger datasets, e.g. extended in time or in three spatial dimensions.

To show the power of these methods, we applied them to a simulation and an OM experiment to find the length and orientation of PDLs over time. Here we conclude that using a method sensitive to PDLs allows to also identify linear rotor cores in experiment. However, having identified linear cores (PDLs) in optical voltage mapping of intact rabbit hearts 13 and human immortalized atrial myocyte cultures (in this work) does not allow to draw general conclusions. Therefore, we propose to use the suggested methods also on other datasets, starting with existing optical voltage mapping results. Then, the presented methods can be used to characterize PDL size and rotation. Such measurement would give another handle to judge the degree to which mathematical models of the heart resemble reality, in addition to e.g. reproducing restitution curves and observing basic spiral dynamics in terms of meander and stability.

The methods used here are available as python scripts from our online repositories, see the data availability statement. Please cite this paper when using the implementation. Note that some methods were originally introduced elsewhere: The AM method 24], the CM method 12 and RPG method [13. The algorithms have currently been tested on dense Cartesian grids in 2D, but can in a natural way be extended to $3 \mathrm{D}$ and time, and unstructured grids (meshes), where the distinction between vertex-based densities $\rho$ and edge-based densities $\sigma$ will play a more prominent role. Further work can also be directed to see how the methods perform under noisy conditions and lower spatial and/or temporal sampling.

\section{Conclusion}

In this work, we demonstrate that in order to visualize phase defects in dense $2 \mathrm{D}$ data, it is recommended to use LAT-based methods or to use the skewed-phase to derive it 
from snapshots. Several algorithms were proposed to highlight the phase defects visually, for which the simple methods (CM, RPG, CPG, PC) were most effective.

We applied the methods to simulations and an optical voltage mapping experiment; in the latter case we found that in a hiAM cell-culture, the average PDL length in a multi-spiral state was $(4.01 \pm 0.55) \mathrm{mm}$ and precession period $T$ was $(169.2 \pm 9.3) \mathrm{ms}$. We made our detection methods publicly available on our institutional repository and hope it can serve to further help understanding the building blocks of cardiac excitation patterns.

\section{Supporting information}

In the supplementary material, we provide figures of phase defect densities $\rho, \sigma$ for the different methods for AP and BOCF reaction kinetics, to enable a full comparison between methods.

The numerical methods implemented for this paper are available as a Python module at https://gitlab.com/heartkor/py_ithildin. The Python scripts used to generate the figures in this paper are available at

https://gitlab.com/heartkor/scripts-pdl-detection. Finally, we have archived the simulation output and pre-processed optical voltage mapping data on which the scripts were applied on Zenodo (DOI: 10.5281/zenodo.5785785). This archive also contains the Python module and scripts.

Please cite this paper when using the implementation and/or the data. 


\section{S1 Fig.}
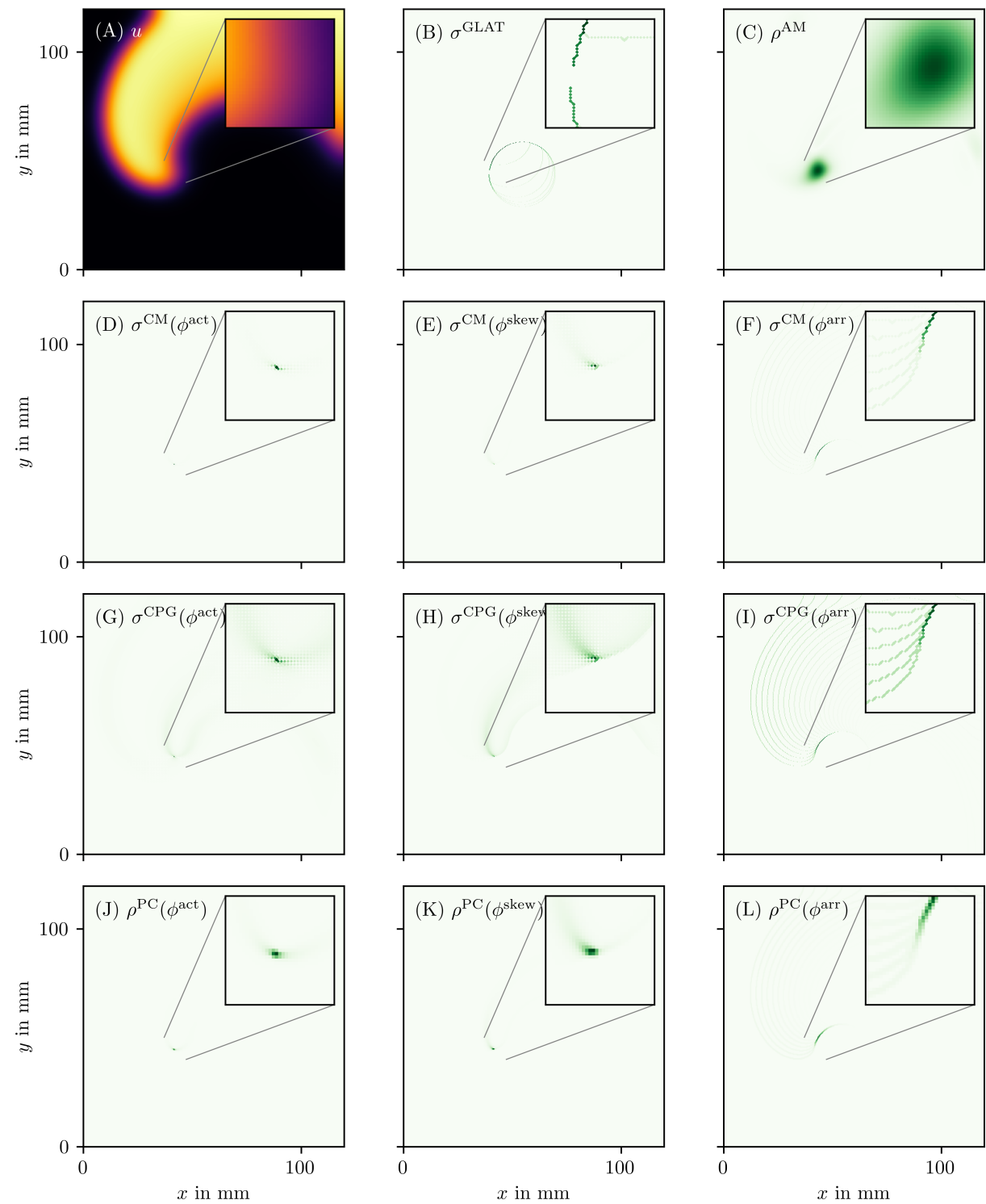

Overview of phase defect detection methods for one snapshot of the AP data set. The data are presented in the same way as in Fig 6 
S2 Fig.
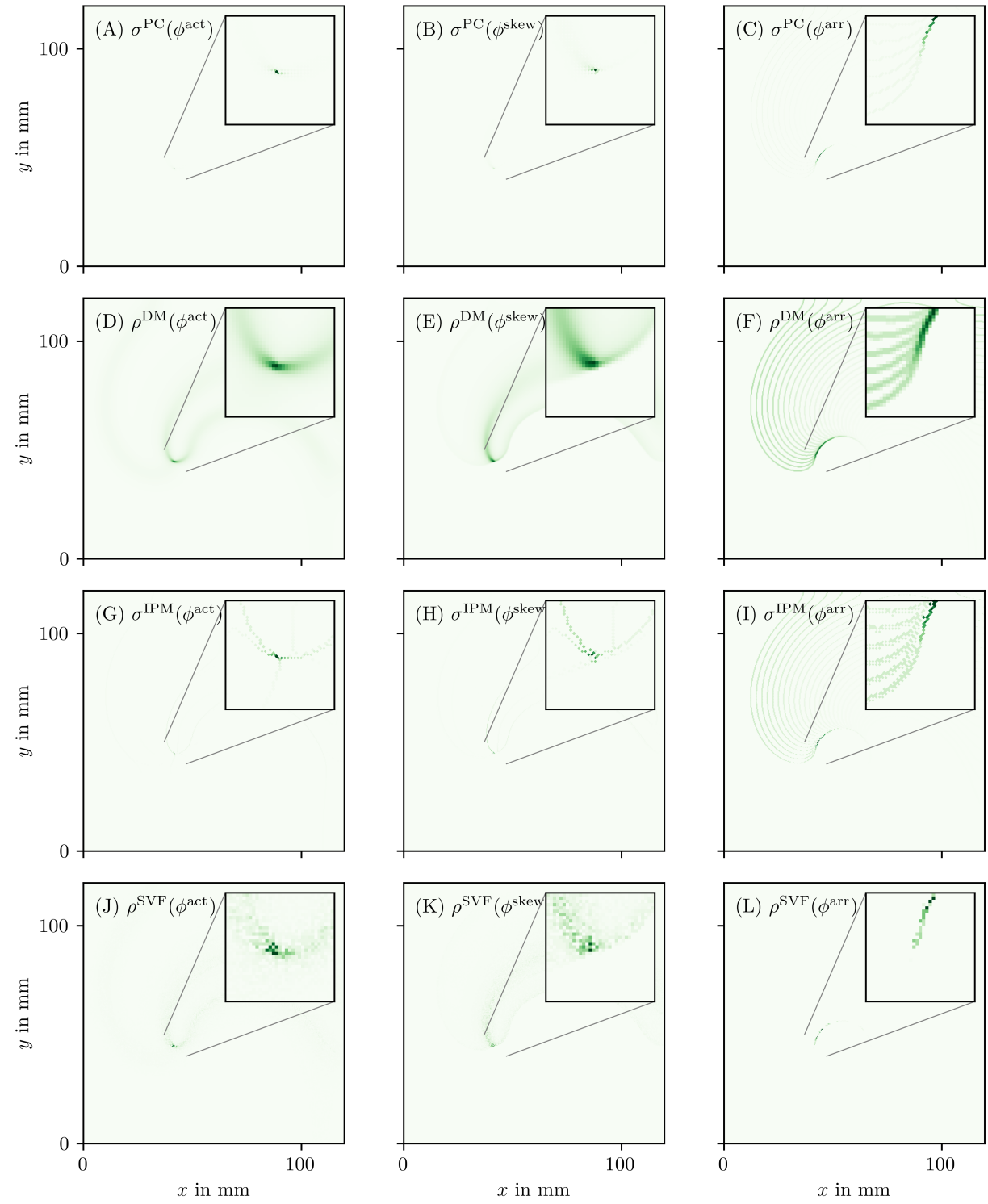

Overview of more phase defect detection methods for one snapshot of the AP data set as in S1 Fig. 


\section{S3 Fig.}
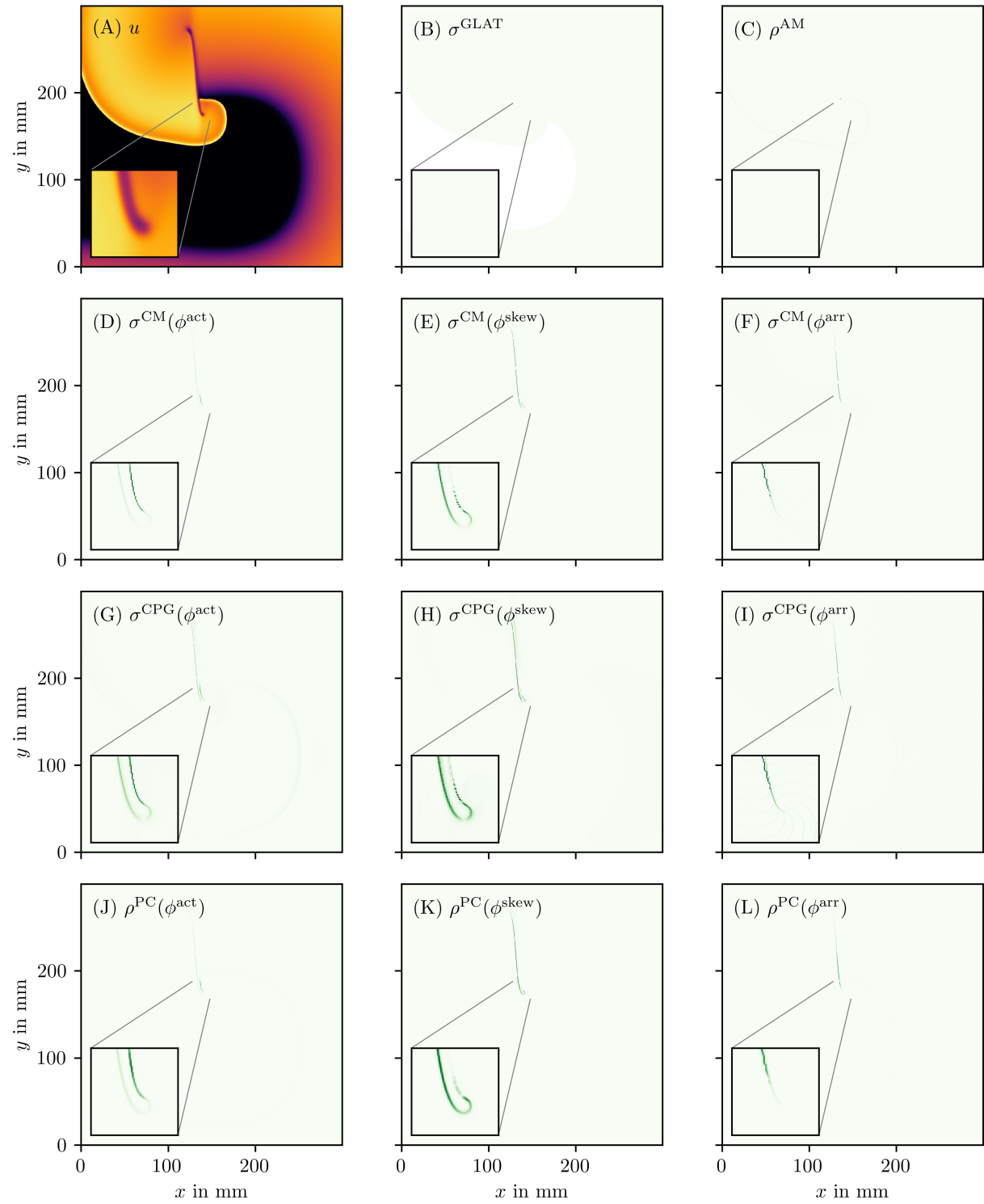

Overview of phase defect detection methods for one snapshot of the BOCF data set. The data are presented in the same way as in Fig 6 


\section{S4 Fig.}
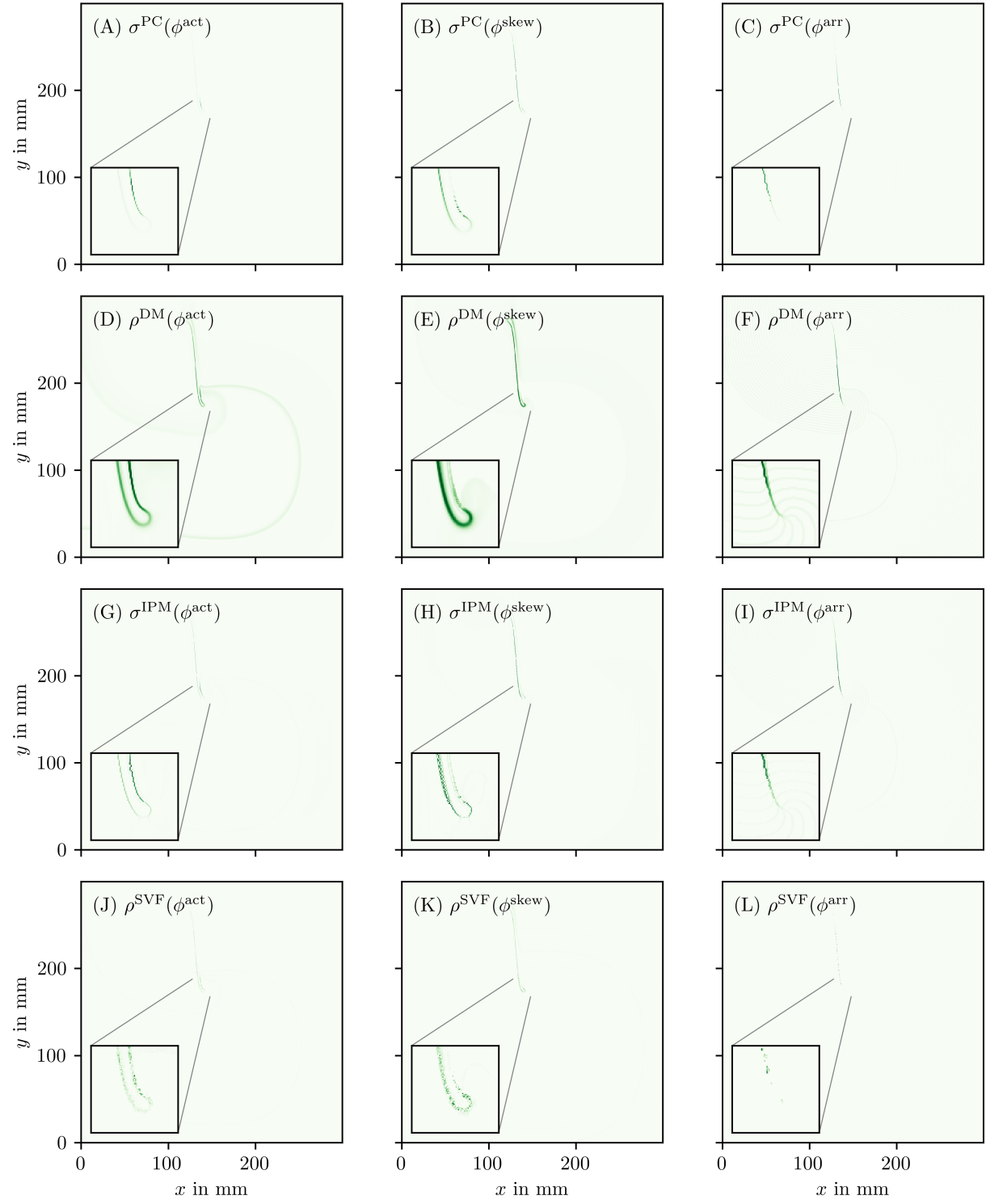

Overview of more phase defect detection methods for one snapshot of the BOCF data set as in S3 Fig.

\section{Acknowledgments}

We are grateful to Sven O. Dekker, Niels Harlaar, Daniël A. Pijnappels and Antoine A.F. de Vries for providing optical voltage mapping data of cardiomyogenically differentiated hiAM monolayers. Moreover, we thank Tim De Coster for helpful comments on the analogy between a PDL and the spiral wave tip trajectory. 


\section{Author contribution}

HD and AVP conceived the study. DK, LA and LL ran the numerical simulations and implemented the detection algorithms. All authors wrote parts of the manuscript and assisted in internal reviewing.

\section{Funding}

DK is supported by KU Leuven grant GPUL/20/012. LA was funded by a KU Leuven FLOF grant and a FWO-Flanders fellowship, grant $1177022 \mathrm{~N}$; LL was funded by KU Leuven and FWO-Flanders, grant G025820N. Research at Sechenov University was financed by the Ministry of Science and Higher Education of the Russian Federation within the framework of state support for the creation and development of World-Class Research Centers "Digital biodesign and personalized healthcare" 075-15-2020-926.

\section{References}

1. Allessie MA, Bonke FIM, Schopman FJG. Circus movement in rabbit atrial muscle as a mechanism of tachycardia. Circ Res. 1973;33:54-62.

2. Gray RA, Pertsov AM, Jalife J. Spatial and temporal organization during cardiac fibrillation. Nature. 1998;392:75-78.

3. Gray RA, Jalife J, Panfilov A, Baxter WT, Cabo C, Pertsov AM. Non-stationary vortex-like reentrant activity as a mechanism of polymorphic ventricular tachycardia in the isolated rabbit heart. Circulation. 1995;91:2454-2469.

4. Zykov VS. Simulation of wave processes in excitable media. Manchester: Manchester University Press; 1987.

5. Fenton FH, Karma A. Vortex dynamics in three-dimensional continuous myocardium with fiber rotation: filament instability and fibrillation. Chaos. $1998 ; 8: 20-47$.

6. Bray M, Wikswo J. Considerations in phase plane analysis for nonstationary reentrant cardiac behavior. Physical Review E. 2002;65(5, 1). doi:10.1103/PhysRevE.65.051902.

7. Winfree AT. Varieties of spiral wave behavior: An experimental approach to the theory of excitable media. Chaos. 1990;1:303-334.

8. Efimov IR, Sidorov VY, Cheng Y, Wollenzier B. Evidence of 3D Scroll Waves with Ribbon-Shaped Filament as a Mechanism of Ventricular Tachycardia in the Isolated Rabbit Heart. J Cardiovasc Electrophysiol. 1999;10:1452-1462.

9. Janse MJ, van Capelle FJL, Morsink H, Kleber AG, Wilms-Schopman FJG, Cardinal R, et al. Flow of injury current and patterns of excitation during early ventricular arrhythmias in acute regional myocardial ischemia in isolated porcine and canine hearts. Evidence for 2 different arrhythmogenic mechanisms. Circulation Research. 1980;47:151-165. 
10. Clayton RH, Zhuchkova EA, Panfilov AV. Phase singularities and filaments: Simplifying complexity in computational models of ventricular fibrillation. Prog Biophys Molec Biol. 2005;90:378-398.

11. Keener JP. The effects of discrete gap junction coupling on propagation in myocardium. JTheor Biol. 1991;148:49-82.

12. Tomii N, Yamazaki M, Ashihara T, Nakazawa K, Shibata N, Honjo H, et al. Spatial phase discontinuity at the center of moving cardiac spiral waves. Computers in Biology and Medicine. 2021;130.

13. Arno L, Quan J, Nguyen NT, Vanmarcke M, Tolkacheva EG, Dierckx H. A Phase Defect Framework for the Analysis of Cardiac Arrhythmia Patterns. Front Physiol. 2021;12. doi:10.3389/fphys.2021.690453.

14. Winfree AT. Patterns of phase compromise in biological cycles. Journal of Mathematical Biology. 1974;1. doi:10.1007/bf02339491.

15. Arno L, Quan J, Vanmarcke M, Nguyen N, Tolkacheva EG, Dierckx H. Phase defect lines during cardiac arrhythmias: from theory to experiment. https://arxivorg/abs/210100315. 2021;

16. Arfken GB, Weber HJ. Mathematical methods for physicists; 4th ed. San Diego, CA: Academic Press; 1995.

17. Clayton RH, Bernus O, Cherry EM, Dierckx H, Fenton FH, Mirabella L, et al. Models of cardiac tissue electrophysiology: Progress, challenges and open questions. Prog Biophys Molec Biol. 2011;104:22-48.

18. Bueno-Orovio A, Cherry EM, Fenton FH. Minimal model for human ventricular action potentials in tissue. J Theor Biol. 2008;253:544-560.

19. Priebe L, Beuckelmann DJ. Simulation study of cellular electric properties in heart failure. CircRes. 1998;82:1206-1223.

20. Aliev RR, Panfilov AV. A simple two-variable model of cardiac excitation. Chaos, Solitons and Fractals. 1996;7:293-301.

21. Harlaar N, Dekker SO, Zhang J, Snabel R, Veldkamp MW, Verkerk AO, et al. Genetic engineering of the first functional human cardiomyocyte lines for building clinically relevant atrial fibrillation models. Nature Biomedical Engineering. 2021;forthcoming.

22. Kuklik P, Zeemering S, Maesen B, Maessen J, Crijns HJ, Verheule S, et al. Reconstruction of instantaneous phase of unipolar atrial contact electrogram using a concept of sinusoidal recomposition and Hilbert transform. IEEE Transactions on Biomedical Engineering. 2015;62:296-302.

23. Kuramoto Y. Chemical Oscillations, Waves, and Turbulence. Berlin: Springer; 1984.

24. Winfree A, Caudle S, Chen G, McGuire P, Szilagyi Z. Quantitative optical tomography of chemical waves and their organizing centers. Chaos: An Interdisciplinary Journal of Nonlinear Science. 1996;6(4):617-626.

25. Hagberg AA, Schult DA, Swart PJ. Exploring Network Structure, Dynamics, and Function using NetworkX. In: Varoquaux G, Vaught T, Millman J, editors. Proceedings of the 7th Python in Science Conference. Pasadena, CA USA; 2008. p. $11-15$. 